\title{
CRISPR/Cas-mediated non-viral genome specific targeted CAR T cells achieve high safety and efficacy in relapsed/refractory B-cell non-Hodgkin lymphoma
}

\section{He Huang ( $\nabla$ huanghe@zju.edu.cn )}

The First Affiliated Hospital, School of Medicine, Zhejiang University https://orcid.org/0000-0002-27231621

Jiqin Zhang

East China Normal University

\section{Yongxian $\mathrm{Hu}$}

The First Affiliated Hospital, School of Medicine, Zhejiang University

\section{Jiaxuan Yang}

Shanghai Research Center for Gene Editing and Cell Therapy, Shanghai Key Laboratory of Regulatory Biology, School of Life Sciences, East China Normal University, Shanghai, China

\section{Wei Li}

Bioray Laboratories Inc., Shanghai, China

\section{Mingming Zhang}

Bone Marrow Transplantation Center, The First Affiliated Hospital, School of Medicine, Zhejiang University, Hangzhou, Zhejiang, China

\section{Yue Tian}

Shanghai Research Center for Gene Editing and Cell Therapy, Shanghai Key Laboratory of Regulatory Biology, School of Life Sciences, East China Normal University, Shanghai, China

\section{Guoqing Wei}

Bone Marrow Transplantation Center, The First Affiliated Hospital, School of Medicine, Zhejiang University, Hangzhou, Zhejiang, China

\section{Linjie Zhang}

Shanghai Research Center for Gene Editing and Cell Therapy, Shanghai Key Laboratory of Regulatory Biology, School of Life Sciences, East China Normal University, Shanghai, China

\section{Kui Zhao}

PETCT Center, The First Affiliated Hospital, School of Medicine, Zhejiang University, Hangzhou, Zhejiang, China

\section{Binghe Tan}

Shanghai Research Center for Gene Editing and Cell Therapy, Shanghai Key Laboratory of Regulatory Biology, School of Life Sciences, East China Normal University, Shanghai, China 


\section{Jiazhen Cui}

Bone Marrow Transplantation Center, The First Affiliated Hospital, School of Medicine, Zhejiang University, Hangzhou, Zhejiang, China

\section{Yalei Qi}

Shanghai Research Center for Gene Editing and Cell Therapy, Shanghai Key Laboratory of Regulatory Biology, School of Life Sciences, East China Normal University, Shanghai, China

\section{Yi Li}

Bone Marrow Transplantation Center, The First Affiliated Hospital, School of Medicine, Zhejiang University, Hangzhou, Zhejiang, China

\section{Qiliang Tian}

Bioray Laboratories Inc., Shanghai, China

\section{Qingcan Wang}

Bioray Laboratories Inc., Shanghai, China

\section{Yuxuan Wu}

Shanghai Research Center for Gene Editing and Cell Therapy, Shanghai Key Laboratory of Regulatory Biology, School of Life Sciences, East China Normal University, Shanghai, China

\section{Dali Li}

Shanghai Key Laboratory of Regulatory Biology East China Normal University https://orcid.org/00000002-0046-8493

\section{Bing Du}

Shanghai Research Center for Gene Editing and Cell Therapy, Shanghai Key Laboratory of Regulatory Biology, School of Life Sciences, East China Normal University, Shanghai, China

\section{Mingyao Liu}

East China Normal University

\section{Biological Sciences - Article}

Keywords: non-Hodgkin lymphoma, chimeric antigen receptor (CAR) T cell therapy

Posted Date: April 3rd, 2021

DOI: https://doi.org/10.21203/rs.3.rs-373651/v1

License: (c) (i) This work is licensed under a Creative Commons Attribution 4.0 International License. Read Full License

Version of Record: A version of this preprint was published at Nature on August 31st, 2022. See the published version at https://doi.org/10.1038/s41586-022-05140-y. 
2 cells achieve high safety and efficacy in relapsed/refractory B-cell

3 non-Hodgkin lymphoma

4 Jiqin Zhang ${ }^{1,3,8}$, Yongxian $\mathrm{Hu}^{2,4,5,6,8}$, Jiaxuan Yang ${ }^{1,8}$, Wei $\mathrm{Li}^{3}$, Mingming Zhang ${ }^{2,4,5,6}$,

5 Yue Tian ${ }^{1}$, Guoqing $\mathrm{Wei}^{2,4,5,6}$, Linjie Zhang ${ }^{1}$, Kui Zhao ${ }^{7}$, Binghe Tan ${ }^{1,3}$, Jiazhen

$6 \mathrm{Cui}^{2,4,5,6}$, Yalei $\mathrm{Qi}^{1}$, Yi Li ${ }^{2,4,5,6}$, Qiliang Tian ${ }^{3}$, Qingcan Wang ${ }^{3}$, Yuxuan $\mathrm{Wu}^{1}$, Dali $\mathrm{Li}^{1}$,

7 Bing $\mathrm{Du}^{1, 凶}$, Mingyao Liu ${ }^{1, 凶}, \mathrm{He}^{\mathrm{Huang}}{ }^{2, 凶}$

8

9

${ }^{1}$ Shanghai Research Center for Gene Editing and Cell Therapy, Shanghai Key Laboratory of Regulatory Biology, Institute of Biomedical Sciences and School of Life Sciences, East China Normal University, Shanghai, China

${ }^{2}$ Bone Marrow Transplantation Center, The First Affiliated Hospital, School of Medicine, Zhejiang University, Hangzhou, Zhejiang, China

${ }^{3}$ Bioray Laboratories Inc., Shanghai, China

${ }^{4}$ Zhejiang Province Engineering Laboratory for Stem Cell and Immunity Therapy, Hangzhou, China;

${ }^{5}$ Institute of Hematology, Zhejiang University, China, Hangzhou, China;

${ }^{6}$ Zhejiang Laboratory for Systems \& Precision Medicine, Zhejiang University Medical Center, Hangzhou, China;

${ }^{7}$ PETCT Center, The First Affiliated Hospital, School of Medicine, Zhejiang University, Hangzhou, Zhejiang, China

${ }^{8}$ These authors contributed equally

凶e-mail: bdu@bio.ecnu.edu.cn; myliu@bio.ecnu.edu.cn; huanghe@zju.edu.cn 


\section{Abstract}

In recent years, chimeric antigen receptor (CAR) $\mathrm{T}$ cell therapy has shown great promise in treating hematological malignancies. However, CAR $\mathrm{T}$ cell therapy currently has several limitations. Here we successfully developed a two-in-one approach to generate non-viral genome specific targeted CAR $\mathrm{T}$ cells through CRISPR/Cas9. Based on the optimized protocol, the feasibility was preliminarily demonstrated by a preclinical study inserting an anti-CD19 CAR cassette into the AAVS1 safe harbor locus. We found that non-viral AAVS1-knockin CAR T cells behave comparably to those conventionally produced by lentivirus. Furthermore, an innovative type of anti-CD19 CAR T cells with $P D 1$-integration was constructed and shows a superior ability to eradicate tumor cells with high PD-L1 expression. In adoptive therapy for relapsed/refractory ( $\mathrm{r} / \mathrm{r})$ aggressive B-cell non-Hodgkin lymphoma (B-NHL), we observed a high rate (87.5\%) of complete remission (CR) and durable responses without serious adverse events in eight patients after treatment. Notably, these enhanced CAR T cells were effective even at a low infusion dose and with a low CAR percentage, which indicated that they have higher potency. No off-target events were found in the infusion product. Single-cell RNA sequencing analysis further validated the advantage of PD1 interference that results in fewer dysfunctional CAR $\mathrm{T}$ cells through this treatment. Collectively, our results demonstrate the outstanding safety and efficacy of non-viral genome specific integrated CAR $\mathrm{T}$ cells, thus providing a revolutionary technology for CAR $\mathrm{T}$ cell therapy.

\section{Introduction}

In recent years, CAR T cell therapy has rapidly developed and shows a great potential in cancer therapy, which is exemplified by the FDA approval of four anti-CD19 CAR $\mathrm{T}$ cell treatments ${ }^{1-5}$. Nevertheless, there still remain some limitations, including the complicated manufacturing process, high production cost, long preparation time and potential safety concerns of current therapies. The use of virus in CAR $\mathrm{T}$ cell production is one area of concern, as the disadvantages include that insertional 
mutagenesis increases the risk of tumor development ${ }^{6,7}$. Furthermore, specific responses to virus-derived DNA tend to impede CAR expression ${ }^{8,9}$ and virus manufacture frequently incurs high costs ${ }^{10}$. Although some strategies, such as using transposon systems ${ }^{11-14}$ and mRNA transduction ${ }^{15,16}$, are being exploited to generate CAR T cells without virus, the low homogeneity of final products caused by random integration and discontinued CAR expression become additional problems. Recently, several studies have shown that CRISPR/Cas9 technology can be applied to generate locus specific integrated CAR $\mathrm{T}$ cells by using an adeno-associated virus (AAV) vector as a template ${ }^{17,18}$. Furthermore, one preferential non-viral strategy was proposed to produce $\mathrm{T}$ cell products with point mutation correction and precise insertion of the TCR element ${ }^{19}$. Thus, in order to simultaneously solve the disadvantages of virus usage and random integration, here we further optimized the conditions and developed non-viral genome specific targeted CAR $\mathrm{T}$ cells through CRISPR/Cas9. The feasibility was preliminarily demonstrated by preclinical experiments using AAVS1-targeted anti-CD19 CAR T cells. Given that blockage of the PD1/PD-L1 pathway by inhibitors or gene editing has been reported to improve the antitumor activity of CAR T cells $\mathrm{s}^{20-23}$, we generated enhanced $P D 1$-integrated anti-CD19 CAR T cells and demonstrated their safety and effectiveness in treating patients with $\mathrm{r} / \mathrm{r}$ B-NHL.

\section{Results}

First, we sought to optimize the protocol for producing non-viral genome specific integrated $\mathrm{T}$ cells. It was found that a homology directed repair (HDR) template, in the form of linear double-stranded DNA (dsDNA), could achieve high recombination efficiency and cell viability (Figure 1a, S1a-c). More viable integrated cells were acquired when electroporation was carried out in stimulated $\mathrm{T}$ cells by applying 800bp homology arms (Figure 1b, c, S1d-g, S2). After confirmation of an optimal protocol, for proof of concept, we first chose to introduce the CAR targeting construct into the AAVS1 safe harbor, which excludes the influence caused by functional endogenous genes, to evaluate whether this approach would affect the 
properties of CAR T cells. An anti-CD19 CAR sequence was constructed, which was comprised of the intracellular domain of 4-1BB and CD3 $\zeta$ (named as 19bbz). The integration efficiency of $19 \mathrm{bbz}$ into $A A V S 1$ was about $10 \%$ (up to $19.80 \%$ ) and the indel percentage ranged from $67 \%$ to $87 \%$ in healthy donor cells (Figure 1d, e, S3a). Also, the integration was unbiased between bulk $\mathrm{CD}^{+}, \mathrm{CD}^{+}$and $\mathrm{CD} 8^{+} \mathrm{T}$ cells (Figure S3b, c). To understand the influence caused by the method per se, we comprehensively compared AAVS1-integrated anti-CD19 CAR T cells (named as AAVS1-19bbz) with lentivirus-produced anti-CD19 CAR $\mathrm{T}$ cells (named as LV-19bbz). Although the electroporation procedure itself led to some cell damage, T cell expansion was not impaired and high cell viability was detected after thorough recovery (Figure S3d-f). Interestingly, electroporation manipulation conferred a growth advantage on $\mathrm{CD}^{+} \mathrm{T}$ cells over $\mathrm{CD}^{+}$cells when compared to lentivirus infection (Figure S3g), which was consistent with a previous study ${ }^{19}$. We observed that AAVS1-19bbz and LV-19bbz cells exhibited comparable cell expansion after tumor cell stimulation (Figure 1f, S3h). Our approach did not change the differentiation of $\mathrm{T}$ cell subsets (Figure S3i). In comparison to untreated $\mathrm{T}$ cells, AAVS1-19bbz cells responded to tumor cells as well as LV-19bbz did, with a little difference in cell marker expression and cytokine secretion (Figure 1g, h). Importantly, like LV-19bbz cells, AAVS1-19bbz cells vigorously eradicated tumor cells in vitro and in vivo (Figure 1i, j, S3j-1). Meanwhile, precise integration of the CAR cassette was validated by Sanger sequencing and non-targeted integration detection (Figure S4). Taken together, these results demonstrate that the strategy to produce non-viral genome specific targeted CAR T cells is feasible.

Due to the well-known inhibition of T-cell effector function through the PD1/PD-L1 pathway, we set out to develop an enhanced type of CAR T cells by integrating an anti-CD19 CAR sequence into the PD1 gene (named as PD1-19bbz). CAR expression was detected in about $20 \%$ (up to $30.3 \%$ ) of healthy donor T cells (Figure 2a, b). A high indel percentage (83\%-93\%) was observed in total T cells from five representative donors (Figure 2c). The impairment of PD1 in PD1-19bbz cells was demonstrated by low PD1 protein expression in $\mathrm{CAR}^{+}$cells after co-culture with 
tumor cells (Figure 2d). PD1-19bbz cells had higher proliferation than LV-19bbz cells after repeated stimulation by PD-L1 expressing Raji cells (Figure 2e). As indicated by other reports ${ }^{24-26}$, PD1 disruption did not affect the elevation of activation markers and cytokine secretion to counteract targeted tumor cells (Figure 2f, g). In contrast to LV-19bbz cells, PD1-19bbz cells showed more robust clearance of PDL1-upregulated tumor cells in vitro and in vivo (Figure 3h-j). Collectively, these data indicate that non-viral $P D 1$-integrated CAR T cells have the potential to more effectively eliminate tumor cells.

Based on our preclinical experimental data, we then proceeded to carry out a phase I clinical trial to evaluate the safety and efficacy of PD1-19bbz cells in treating patients with r/r B-NHL (ClinicalTrials.gov NCT04213469). In the final infusion products of eight patients, the average percentages of CAR integration and PDI indel were about $20 \%$ and $60 \%$, respectively (Figure S5a-d). The infusion products had a cell viability of more than $90 \%$, and responded to and eradicated tumor target cells in vitro (Figure S5e-g). Next, we undertook whole genome sequencing (WGS) to detect off-target events in one representative infusion product. Exclusive indels in the edited sample, which located around 2,219 potential off-target sites predicted by Cas-OFFinder, were identified by bioinformatics and further validated by deep sequencing. As a result, no verified indels were found within $200 \mathrm{bp}$ upstream and downstream of these sites (Figure S6, Table S1). Indel events were also not detected at 29 top-ranked potential off-target sites predicted by the Benchling CRISPR tool, by using deep sequencing analysis (Table S2).

Eight patients were given a lymphodepleting chemotherapy regimen using combined cyclophosphamide and fludarabine, followed by one infusion of PD1-19bbz cells with a dose of $0.56 \times 10^{6}-2.35 \times 10^{6}$ cells $/ \mathrm{kg}$ body weight (Table $1, \mathrm{~S} 3, \mathrm{~S} 4$ ). While all the patients experienced transient and reversible hematologic toxicity events mainly related to the chemotherapy pretreatment, no other high-grade $(\geq 3)$ adverse events were found (Table S5). Mild cytokine release syndrome (CRS) was observed in some patients and no immune effector cell-associated neurotoxicity syndrome (ICANS) occurred (Figure 3a, S7). PD1-19bbz cells proliferated and persisted in vivo 
(Figure 3b, c). While the peak of CAR T cell expansion in most patients was on day 7 to 14 after infusion, a slower changing curve was detected in one patient. During a median observation period of five months, CR was achieved in $7 / 8(87.5 \%)$ patients as shown by positron emission tomography-computed tomography (PET-CT) scans and durable responses were found in all seven patients at the time of last follow-up (Figure 3d, e, Table 1). Partial remission (PR) was observed in the remaining (1/8) patient, thus the best objective response rate reached $100 \%$ in all the patients. Of note, PD1-19bbz cells effectively functioned even at a low infusion dose and with a low CAR percentage, thereby indicating high potency of these PDI knockout CAR T cells. Together, these data demonstrate that non-viral PDI-integrated CAR T cells have high safety and efficacy for patients with r/r B-NHL.

To further understand the characteristics of non-viral $P D 1$-integrated CAR T cells before and after infusion, single-cell RNA sequencing (scRNA-seq) was carried out in three patient samples. After standard data processing and quality control procedures, transcriptomic profiles of 54,774 cells were obtained. Total cells were divided into five types using a graph-based clustering method (Figure S8) and the subtype of $\mathrm{CD}^{+} \mathrm{T}$ cells was further analyzed (Figure S9). To unravel the features of infusion products, two clusters $(\mathrm{C} 1, \mathrm{C} 2)$ were defined by using $\mathrm{CD} 8$ memory and dysfunction marker genes, and the expression of a wide range of memory, dysfunction and cytotoxicity genes ${ }^{27-29}$ was analyzed (Figure 4a, S10b-h, Table S6). It was noteworthy that the percentage of the CD8 memory cluster was about $80 \%$ in mixed samples, and it even reached above $95 \%$ in two individual samples (Figure $4 \mathrm{~b}$ ). The proportion of $\mathrm{C} 1$ and $\mathrm{C} 2$ was similar between $\mathrm{CAR}^{+}$and $\mathrm{CAR}^{-}$cells (Figure S10a, S11). Next, we set out to understand the kinetics of gene expression in $\mathrm{CD}^{+} / \mathrm{CAR}^{+}$ cells through the treatment (Figure 4c). As expected, almost no expression of PD1 was detected in all the samples (Figure 4d, S10i). Intriguingly, like PD1, a series of dysfunction genes including BTLA, CD244, CD200, CD109, ENTPD1, LAYN and CXCL13 maintained very low expression as well (Figure 4d, Table S7-S9), which was in line with the previous findings ${ }^{30,31}$. Additionally, sustained expression of memory genes and attenuated expression of dysfunction genes were found in $\mathrm{CAR}^{+}$ 
cells after infusion into patients (Figure 4e-f, S12, S13, Table S7-S9), thereby suggesting that PD1-19bbz cells had a lower tendency to become exhausted in vivo. The activities of different pathways were also analyzed in the samples (Figure S14). Altogether, these scRNA-seq data reveal more memory and fewer dysfunctional $\mathrm{CAR}^{+}$cells in pre-infused and post-infused PD1-19bbz cells, thus giving a mechanistic explanation for their superior efficacy in the clinical trial.

\section{Discussion}

CRISPR/Cas9-mediated HDR is becoming a usual method to facilitate precise integration of target sequences ${ }^{32-34}$. Recently, one study showed the feasibility of editing human $\mathrm{T}$ cells using a non-viral genome targeting strategy ${ }^{19}$. Here, we further optimized the protocol to achieve higher recombination efficiency and thus generated genome specific targeted CAR T cells without using virus. Despite a relatively low percentage of $\mathrm{CAR}^{+}$cells in comparison to lentivirus-produced CAR T cells, we substantiate that non-viral genome specific integrated CAR T cells are effective and an additional enrichment step is unnecessary for clinical application. In addition, although the electroporation step results in some cell damage, our data indicate that $\mathrm{T}$ cell expansion ability is not impaired and the cell number and viability of the final product can fully meet the requirements for clinical treatment. In accordance with the concept that using ribonucleoproteins (RNPs) can reduce the off-target risk ${ }^{35,36}$, we have not, indeed, found any indel events using WGS and deep sequencing analyses, thus mitigating the safety concern of genome editing. During the process from bench to production, an unexpected lower CAR recombination efficiency in two infusion products (patient-1, patient-4) and low PD1 indel percentage in one infusion product (patient-1) were detected. The reason is attributed to the early premature manufacturing process, which has been solved, rather than individual variance or low reproducibility of method. Taken together, we demonstrate the feasibility of formal large-scale production of non-viral genome specific targeted CAR T cells for clinical application.

We are the first to demonstrate the safety and efficacy of non-viral genome 
specific targeted CAR T cells in a clinical trial. Relative to conventional CAR T cell therapies $^{37-39}$, we found superior safety for patients with r/r B-NHL by using non-viral PD1-integrated anti-CD19 CAR T cells, with only a low percentage of mild CRS and without occurrence of neurologic toxicity. Our results are also consistent with two recently reported clinical trials ${ }^{40,41}$ and accordingly further demonstrate the safety of CRISPR/Cas9 application in T cell therapy. It was reported that the rates of $\mathrm{CR}$ in treating patients with $\mathrm{r} / \mathrm{r}$ B-NHL by three FDA approved autologous anti-CD19 CAR $\mathrm{T}$ cell therapies were $58 \%{ }^{37}, 40 \%{ }^{38}$ and $53 \%{ }^{39}$, respectively. In contrast, we observed a striking high rate $(87.5 \%)$ of CR. Surprisingly, despite an unexpectedly low initial dose or simultaneous low CAR percentage, CR was achieved in all three patients, which indicates that non-viral PD1-targeted CAR T cells indeed have more potency to kill tumor cells. Thus, it prompts us to test lower infusion doses in future clinical trials, which may further reduce the preparation time and production cost.

The outstanding clinical efficacy can be explained by our scRNA-seq data in two aspects. First, when compared to conventional CAR T cells produced by lentivirus ${ }^{29}$, there was a much higher percentage of $\mathrm{CD}^{+}$memory cells in the infusion products of non-viral $P D 1$-integrated CAR T cells. Second, driven by the loss of PD1, the in vivo persistence of fewer dysfunctional CAR T cells was found through the treatment. Additionally, our results revealed that PD1 knockout causes the downregulation of diverse immune suppressive signaling in CAR $\mathrm{T}$ cells, which suggests that gene editing strategy may have an advantage over those that only abolish the interaction between PD1 and PD-L1 by using antibodies. Given that inhibitory receptors which parallel PD1 function, such as LAG3, TIM3 and TIGIT, were still highly expressed in $\mathrm{CAR}^{+}$cells according to our data (Figure 4c, S13b, Table S7-S9), simultaneously intervening in multiple pathways holds promise to further augment the function of CAR T cells. In conclusion, our clinical results give solid evidence for the real advantage of non-viral genome specific integrated CAR T cells, thus uncovering its great potential in treating more malignancies, especially solid tumors, in the future.

In this study, we describe an innovative strategy to develop non-viral genome specific targeted CAR T cells by CRISPR/Cas9. This technology is advanced due to 
combining the advantages of both non-viral manufacturing processes and precise genome editing. As a two-in-one approach without using virus, the manufacturing procedure is simplified, with shortened preparation time, reduced production expenses, and increased safety and efficacy of CAR $\mathrm{T}$ cell products. These advantages are significant, especially for the generation of gene modified CAR T cells where virus preparation and genome editing process are normally both required. On the other hand, locus-specific integration augments the homogeneity of CAR T cells and makes it possible to exploit versatile cell products. Importantly, for the first time, we show the feasible application of this technology from bench to bedside and demonstrate its remarkable safety and efficacy in a clinical trial. Thus, we propose a revolutionary CAR T technology to break through the current barriers and show the considerable potential of CRISPR/Cas9-mediated non-viral genome specific targeted technology in cell therapy.

\section{References}

1 Larson, R. C. \& Maus, M. V. Recent advances and discoveries in the mechanisms and functions of CAR T cells. Nat Rev Cancer 21, 145-161 (2021).

2 MacKay, M. et al. The therapeutic landscape for cells engineered with chimeric antigen receptors. Nat Biotechnol 38, 233-244 (2020).

3 June, C. H., O'Connor, R. S., Kawalekar, O. U., Ghassemi, S. \& Milone, M. C. CAR T cell immunotherapy for human cancer. Science 359, 1361-1365 (2018).

4 June, C. H. \& Sadelain, M. Chimeric Antigen Receptor Therapy. N Engl J Med 379, 64-73 (2018).

5 Labanieh, L., Majzner, R. G. \& Mackall, C. L. Programming CAR-T cells to kill cancer. Nature Biomedical Engineering 2, 377-391 (2018).

6 Michieletto, D., Lusic, M., Marenduzzo, D. \& Orlandini, E. Physical principles of retroviral integration in the human genome. Nat Commun 10, 575 (2019).

7 Russo-Carbolante, E. M. D. et al. Integration pattern of HIV-1 based lentiviral vector carrying recombinant coagulation factor VIII in Sk-Hep and 293T cells. Biotechnology Letters 33, 23-31 (2011).

8 Atianand, M. K. \& Fitzgerald, K. A. Molecular basis of DNA recognition in the immune system. $J$ Immunol 190, 1911-1918 (2013).

9 Tao, J. L., Zhou, X. \& Jiang, Z. F. cGAS-cGAMP-STING: The Three Musketeers of Cytosolic DNA Sensing and Signaling. lubmb Life 68, 858-870 (2016).

10 Gandara, C., Affleck, V. \& Stoll, E. A. Manufacture of Third-Generation Lentivirus for Preclinical Use, with Process Development Considerations for Translation to Good Manufacturing Practice. Human Gene Therapy Methods 29, 1-15 (2018). 
Hurton, L. V. et al. Tethered IL-15 augments antitumor activity and promotes a stem-cell memory subset in tumor-specific T cells. Proceedings of the National Academy of Sciences of the United States of America 113, E7788-E7797 (2016).

Kebriaei, P. et al. Phase I trials using Sleeping Beauty to generate CD19-specific CAR T cells. Journal of Clinical Investigation 126, 3363-3376 (2016).

Maiti, S. N. et al. Sleeping Beauty System to Redirect T-cell Specificity for Human Applications. Journal of Immunotherapy 36, 112-123 (2013).

Monjezi, R. et al. Enhanced CAR T-cell engineering using non-viral Sleeping Beauty transposition from minicircle vectors. Leukemia 31, 186-194 (2017).

Foster, J. B. et al. Purification of mRNA Encoding Chimeric Antigen Receptor Is Critical for Generation of a Robust T-Cell Response. Human Gene Therapy 30, 168-178 (2019).

Lin, L. et al. Preclinical evaluation of CD8+anti-BCMA mRNA CAR T cells for treatment of multiple myeloma. Leukemia (2020).

Eyquem, J. et al. Targeting a CAR to the TRAC locus with CRISPR/Cas9 enhances tumour rejection. Nature 543, 113-+ (2017).

Dai, X. Y. et al. One-step generation of modular CAR-T cells with AAV-Cpf1. Nat Methods 16, 247-+ (2019).

Roth, T. L. et al. Reprogramming human T cell function and specificity with non-viral genome targeting. Nature 559, 405-409 (2018).

Cherkassky, L. et al. Human CAR T cells with cell-intrinsic PD-1 checkpoint blockade resist tumor-mediated inhibition. Journal of Clinical Investigation 126, 3130-3144 (2016).

John, L. B. et al. Anti-PD-1 Antibody Therapy Potently Enhances the Eradication of Established Tumors By Gene-Modified T Cells. Clinical Cancer Research 19, 5636-5646 (2013).

Rafiq, S. et al. Targeted delivery of a PD-1-blocking scFv by CAR-T cells enhances anti-tumor efficacy in vivo. Nat Biotechnol 36, 847-856 (2018).

Ren, J. T. et al. Multiplex Genome Editing to Generate Universal CAR T Cells Resistant to PD1 Inhibition. Clinical Cancer Research 23, 2255-2266 (2017).

Rupp, L. J. et al. CRISPR/Cas9-mediated PD-1 disruption enhances anti-tumor efficacy of human chimeric antigen receptor T cells. Sci Rep 7, 737 (2017).

Su, S. et al. CRISPR-Cas9 mediated efficient PD-1 disruption on human primary T cells from cancer patients. Sci Rep 6, 20070 (2016).

Guo, X. et al. Disruption of PD-1 Enhanced the Anti-tumor Activity of Chimeric Antigen Receptor T Cells Against Hepatocellular Carcinoma. Front Pharmacol 9, 1118 (2018).

van der Leun, A. M., Thommen, D. S. \& Schumacher, T. N. CD8(+) T cell states in human cancer: insights from single-cell analysis. Nature Reviews Cancer 20, 218-232 (2020).

Sade-Feldman, M. et al. Defining T Cell States Associated with Response to Checkpoint Immunotherapy in Melanoma. Cell 175, 998-+ (2018).

Deng, Q. et al. Characteristics of anti-CD19 CAR T cell infusion products associated with efficacy and toxicity in patients with large B cell lymphomas. Nat Med 26 (2020). with predictive potential in non-small-cell lung cancer treated with PD-1 blockade. Nat Med 24, 994-+ (2018).

31 Zheng, C. H. et al. Landscape of Infiltrating T Cells in Liver Cancer Revealed by Single-Cell Sequencing. Cell 169, 1342-+ (2017). 

(2020).

33 Hsu, P. D., Lander, E. S. \& Zhang, F. Development and Applications of CRISPR-Cas9 for Genome Engineering. Cell 157, 1262-1278 (2014).

Komor, A. C., Badran, A. H. \& Liu, D. R. CRISPR-Based Technologies for the Manipulation of Eukaryotic Genomes. Cell 168, 20-36 (2017).

Liang, X. Q. et al. Rapid and highly efficient mammalian cell engineering via Cas 9 protein transfection. J Biotechnol 208, 44-53 (2015). Kim, S., Kim, D., Cho, S. W., Kim, J. \& Kim, J. S. Highly efficient RNA-guided genome editing in human cells via delivery of purified Cas9 ribonucleoproteins. Genome Res 24, 1012-1019 (2014).

Locke, F. L. et al. Long-term safety and activity of axicabtagene ciloleucel in refractory large B-cell lymphoma (ZUMA-1): a single-arm, multicentre, phase 1-2 trial. Lancet Oncol 20, 31-42 (2019). Schuster, S. J. et al. Tisagenlecleucel in Adult Relapsed or Refractory Diffuse Large B-Cell Lymphoma. N Engl J Med 380, 45-56 (2019).

Abramson, J. S. et al. Lisocabtagene maraleucel for patients with relapsed or refractory large B-cell lymphomas (TRANSCEND NHL 001): a multicentre seamless design study. Lancet 396, 839-852 (2020).

Lu, Y. et al. Safety and feasibility of CRISPR-edited T cells in patients with refractory non-small-cell lung cancer. Nat Med 26, 732-740 (2020).

Stadtmauer, E. A. et al. CRISPR-engineered T cells in patients with refractory cancer. Science 367, 1001-+ (2020).

\section{Clinical trial information and design}


safety and efficacy of non-viral PD1-integrated anti-CD19 CAR T cells in treating relapsed/refractory (r/r) aggressive B-cell non-Hodgkin lymphoma (B-NHL). The clinical protocol has been registered at ClinicalTrials.gov (NCT04213469). The inclusion criteria were as follows: 1) aged 18 to 70 years old; 2) diagnosed with CD19 positive r/r B-NHL (stage III-IV); 3) life expectancy of $>3$ months; 4) with Eastern Cooperative Oncology Group (ECOG) score of $\leq 2$ and satisfactory major organ functions; 5) a negative pregnancy test for women of reproductive potential and agreement of using birth control during the study. The exclusion criteria included: 1) pregnancy or breast feeding women; 2) refusal to use birth control during the next two years; 3) underwent allo-HSCT within six months or previous treatment of graft versus host disease; 4) active autoimmune disease which requires immunosuppressive agents; 5) active infection; 6) history of other malignances; 7) ineligibility or lack of ability to comply with the study. In order to preliminarily assess the safety and effectiveness of this novel CAR T cell therapy, eight patients were enrolled in the cohort with infusion dose of $2 \times 10^{6} \mathrm{CAR} \mathrm{T}$ cells/kg. Due to the premature manufacturing process and individual variance, the cell number of three infusion products could not meet the planned dose requirement, thus the actual infusion doses in these patients were lower than $1 \times 10^{6} / \mathrm{kg}$ (Table $1, \mathrm{~S} 3$ ). This therapy included 3 days of lymphodepletion chemotherapy using combined fludarabine $(25 \mathrm{mg} / \mathrm{m} 2$ from day -4 to -2 ) and cyclophosphamide ( $250 \mathrm{mg} / \mathrm{m} 2$ from day -3 to -2$)$. CAR T cell infusion was performed 2 days after the end of lymphodepletion chemotherapy and was followed by standard monitoring. All patients provided written informed consent in accordance with the Declaration of Helsinki before enrolment in the study. The clinical protocol was reviewed and approved by the Clinical Research Ethics Committee of the First Affiliated Hospital, College of Medicine, Zhejiang University. Characteristics, clinical responses and prior therapies of the patients are shown in Table 1 and Table S4. Non-viral PD1-targeted CAR T cells for clinical treatment were manufactured by Bioray Laboratories Inc.

\section{Response assessment}


Treatment response was assessed according to a revised criteria of the Lugano

classification. PET-CT scans and bone marrow biopsy were the major methods applied to evaluate the lymphoma lesions. The response assessment criteria were as follows: 1) CR (complete remission): absence of clinical symptoms, PET-CT and bone marrow evidence associated with lymphoma; 2) PR (partial remission): lymphoma volume decreases at least $50 \%$ without new lymphoma lesions or sustained bone marrow involvement; 3 ) PD (progressive disease): lymphoma volume increases at least $50 \%$ or onset of new lymphoma lesions; 4) SD (stable disease): a condition achieving the criteria for none of CR, PR or PD. The response duration was calculated from the first documentation of response, until progression, initiation of off-study treatment or the last documentation of ongoing response.

\section{Assessment and grading of cytokine release syndrome}

Serum cytokines including IL-2, IL-4, IL-6, IL-10, IFN- $\gamma$, TNF- $\alpha$ and IL-17A were assessed by Human Th1/Th2/Th17 CBA Kit (BD Biosciences) within one month after infusion. Cytokine release syndrome (CRS) was assessed and graded according to the National Cancer Institute Common Terminology Criteria for Adverse Events (NCI-CTCAE) version 5.0 in combination with other methods ${ }^{42}$.

\section{Assessment and grading of neurological toxicity}

Neurological toxicities were assessed and graded according to CTCAE version 5.0. Once CRS symptoms such as pyrexia, hypotension and capillary leak, or other types of adverse events (AEs) were observed, the patient would be closely monitored for signs of neurological toxicity, such as seizure, tremor, encephalopathy and dysphasia.

\section{Assessment and grading of adverse events}

Patients were inpatients and closely monitored after receiving lymphodepletion chemotherapy and CAR T cell infusion. Physical and clinical laboratory examinations were documented during hospitalization to evaluate the toxicity of the treatment. AEs were graded using CTCAE version 5.0. All AEs are summarized in Table S5. During 
hospitalization, any AEs that occurred after CAR $\mathrm{T}$ cell infusion were recorded. Severe AEs, except the decrease of lymphocyte counts caused by lymphodepletion chemotherapy, were required to be reported to the Medical Ethics Committee of the First Affiliated Hospital, College of Medicine, Zhejiang University within 24 hours of the occurrence. One month after infusion, patients were followed up and monitored for disease progression and toxicity once a month.

\section{Cell lines}

Nalm-6 and Raji cells were purchased from ATCC and maintained in RPMI1640 medium (ThermoFisher) supplemented with 10\% fetal bovine serum (ThermoFisher). A Raji cell line stably expressing firefly luciferase (ffLuc) was established by lentivirus infection. Raji cells stably expressing PD-L1 were generated using a lentivirus vector containing a co-expression cassette for PD-L1 and ffLuc. All the stable cell lines underwent selection with puromycine.

\section{Isolation and expansion of human primary $T$ cells}

Fresh peripheral blood mononuclear cells (PBMCs) from healthy donors were provided by Shanghai SAILY Biological Technology Co., Ltd. Fresh PBMCs from patients were collected by apheresis. PBMCs were isolated by density gradient centrifugation using Ficoll (Sigma-Aldrich). T cells were enriched via magnetic separation using anti-CD8/CD4 microbeads (Miltenyi Biotech) and activated with $\mathrm{T}$ Cell TransAct (Miltenyi Biotech). T cells were cultured in X-VIVO media (Lonza) supplemented with $2 \%$ human AB serum or CTS ${ }^{\text {TM }}$ Immune Cell Serum Replacement (ThermoFisher) and recombinant human IL-2 (100 units/mL), IL-7 (5 ng/mL) and IL-15 (5 ng/mL). Cells were harvested once the number reached the requirement for administration, and then washed, formulated and cryopreserved.

\section{CAR T cell generation by lentivirus}

The Anti-CD19 CAR cassette was composed of humanized single-chain variable fragment (scFv) derived from clone FMC63, the extracellular domain and 
transmembrane regions of CD8 $\alpha$, the intracellular domain of 4-1BB (CD137), and the intracellular domain of $\mathrm{CD} 3 \zeta$. The $\mathrm{CAR}$ sequence was cloned into the $\mathrm{pCDH}$ lentiviral vector backbone containing an EF1 $\alpha$ promoter. Lentiviruses were produced by transfecting $293 \mathrm{~T}$ cells with CAR plasmid, pMD2.G and psPAX2 using polyethylenimine (PEI). Virus supernatants were harvested after 3 days to infect primary human $\mathrm{T}$ cells.

\section{RNP and linear double-stranded DNA production}

One two-component single guide RNA (sgRNA) targeting AAVS1 (5'-AGAGCUAGCACAGACUAGAG-3') or PD1 (5'CGACUGGCCAGGGCGCCUGU-3') was chemically synthesized (GenScript) and resuspended with TE buffer. Ribonucleoproteins (RNPs) were produced by complexing AAVS1 or PD1 sgRNA and recombinant spCas9 (ThermoFisher) for 10 minutes at room temperature. RNPs were subjected to electroporation immediately after complex formation. For linear double-stranded DNA (dsDNA) production in preclinical experiments, plasmids containing an mTurquoise 2 or anti-CD19 CAR sequence flanked by homology arms were first constructed. The linear dsDNA was then obtained by restriction endonuclease digestion and purified by TIANgel DNA Purification Kit (Tiangen Biotech).

\section{Human primary $\mathbf{T}$ cell electroporation}

Electroporation was performed 2-3 days after $\mathrm{T}$ cell stimulation. The procedure was conducted following the manufacturer's instructions using a Lonza 4D electroporation system. Briefly, pre-washed $\mathrm{T}$ cells were resuspended in the electroporation buffer P3. Meanwhile, RNPs were prepared followed by mixture with the DNA template. Cells in electroporation buffer were then added and moved into electroporation cuvettes. The program of EO115 was chosen for electroporation. After electroporation, pre-warmed media was immediately supplemented and cells were transferred away from electroporation cuvettes. 
Indel percentage analysis

Genomic DNA was obtained using a Genomic DNA Purification Kit

488

489

490

491

492

493

494

495

496

497

498

499

500

501

502

503

504

505

506

507

508

509

510

511

512

513

514

515

(ThermoFisher). The fragments containing indel sites were amplified by PCR using specific primers and purified by TIANgel DNA Purification Kit (Tiangen Biotech). DNA sequencing was carried out and indel percentage was measured by ICE analysis (Synthego). The primers used were as follows: AAVS1-Forward 5'-CACCACGTGATGTCCTCTGA-3';

AAVS1-Reverse

5'-CCGGCCCTGGGAATATAAGG-3';

PD1-Forward

5'-CCACGTGGATGTGGAGGAAG-3';

PD1-Reverse:

5'-CCACACAGCTCAGGGTAAGG-3'.

\section{Deep Sequencing}

Deep sequencing was carried out to detect indels at 29 top-ranked off-target sites predicted by the Benchling CRISPR tool or to validate the possible indels preliminarily indicated by whole genome sequencing in one representative infusion product (patient-2). Genomic DNA of untreated T cells and infusion products was harvested using Genomic DNA Purification Kit (ThermoFisher). The fragments containing indel sites were amplified by PCR using specific primers and subjected to sequencing on a Hi-TOM platform with $10000 \times$ coverage as described previously ${ }^{43}$.

\section{Whole Genome Sequencing}

Genomic DNA of untreated T cells and the infusion product of patient-2 was extracted using Blood \& Cell Culture DNA Kit (Qiagen) according to the manufacturer's instructions and subjected to library construction. Sequencing libraries were generated using Truseq Nano DNA HT Sample preparation Kit (Illumina) following the manufacturer's recommendations and index codes were added to attribute sequences to each sample. These libraries including untreated and edited $\mathrm{T}$ cells were sequenced on the HiSeq platform (Illumina) with $100 \times$ coverage. BWA (Burrows-Wheeler Aligner) ${ }^{44}$ was used to align the clean reads of each sample against the reference genome (settings: mem -t $5-\mathrm{M}-\mathrm{R}$ ). Alignment files were converted to 
BAM files using SAMtools software ${ }^{45}$ (settings: -bS -t). In addition, potential PCR duplications were removed using the sambamba command "markdup". If multiple read pairs have identical external coordinates, only the pair with the highest mapping quality was retained. Insertions and deletions (indels) $(<50 \mathrm{bp})$ were calculated and identified with MPILEUP in SAMTOOLS ${ }^{45}$. In order to reduce the indel detection error rate, we filtered the indels in which the supported reads number was less than 4 and quality value (MQ) was less than 30 and QUAL was less than 20. Indels were filtered, with those near other variants and within the PAR being removed. The whole genome sequencing was carried out by Novogene Co., Ltd.

We used Cas-OFFinder (http://www.rgenome.net/cas-offinder/) to predict potential off-target sites. Any sequence, followed by an NRG PAM, having no more than five mismatches (a bulge penalty equals two base mismatches) with PD1 sgRNA, was screened and in total 2,219 sites (not including those around on-target site) were identified. Indels exclusively detected in the edited sample and located around potential off-target sites were searched. No indel events were found within $15 \mathrm{bp}$ upstream and downstream $( \pm 15 \mathrm{bp})$ of the sites. Indel events were detected within $200 \mathrm{bp}$ upstream and downstream $( \pm 200 \mathrm{bp})$ of 8 sites. Deep sequencing with 10000 $\times$ coverage was performed to validate these indel events.

\section{Single-cell RNA sequencing}

Fresh PBMCs from patients were collected by apheresis at the peak (D7 or D12) and stable (D28 or D29) stages of CAR T cell expansion after infusion, respectively, and then isolated by density gradient centrifugation using Ficoll (Sigma-Aldrich). Infusion products and PBMCs of three patients (patient-1, patient-2, patient-3) were subjected to single-cell RNA sequencing (scRNA-seq).

The scRNA-seq libraries were generated using the 10X Genomics Chromium Controller Instrument and Chromium Single Cell 3' V3.1 Reagent Kits (10 $\times$ Genomics). Briefly, cells were concentrated to 1000 cells $/ \mu \mathrm{L}$ and approximately 7,000 cells were loaded into each channel to generate single-cell Gel Bead-In-Emulsions (GEMs), which results in mRNA barcoding of an expected 5,000 single-cells for each sample. After the RT step, GEMs were broken and barcoded-cDNA was purified and amplified. The amplified barcoded cDNA was fragmented, A-tailed, ligated with adaptors and index PCR amplified. The final libraries were quantified using the Qubit 
High Sensitivity DNA assay (ThermoFisher) and the size distribution of the libraries was determined using a High Sensitivity DNA chip on a Bioanalyzer 2200 (Agilent). All libraries were sequenced by an Illumina sequencer (Illumina) on a 150bp paired-end run.

We applied fastp ${ }^{46}$ with default parameter filtering of the adaptor sequence and removed the low quality reads to achieve clean data. Then the feature-barcode matrices were obtained by aligning reads to the human genome (GRCh38 Ensemble: version 91) using CellRanger v3.1.0. We applied the down sample analysis among samples sequenced according to the mapped barcoded reads per cell of each sample and finally achieved the aggregated matrix. Cells containing over 200 expressed genes and a mitochondria UMI rate below $20 \%$ passed the cell quality filtering and mitochondrial genes were removed in the expression table.

Seurat package (version: 3.1.4, https://satijalab.org/seurat/) was used for cell normalization and regression based on the expression table according to the UMI counts of each sample and percent of mitochondria rate to obtain the scaled data. PCA was performed based on the scaled data with the top 2000 highly variable genes and the top 10 principals used for tSNE construction and UMAP construction. Utilizing the graph-based cluster method, we acquired the unsupervised cell cluster result based the PCA top 10 principals and we calculated the marker genes by the FindAllMarkers function with the Wilcox rank sum test algorithm under the following criteria: 1) $\operatorname{lnFC}>0.25 ; 2)$ pvalue $<0.05 ; 3)$ min.pct $>0.1$. To characterize the relative activation of a given gene set such as KEGG pathway, Memory, Dysfunction and Cytotoxicity as described previously, we used QuSAGE ${ }^{47}$ (2.16.1) to calculate the score for each cluster/sample and GSVA ${ }^{48}(1.32 .0)$ to calculate it for each cell. ScRNA-seq and data analysis were performed by NovelBio Bio-Pharm Technology Co., Ltd.

\section{Flow cytometry}

CAR and membrane protein expression was determined by flow cytometry. Cells were pre-washed and incubated with antibodies for 30 minutes on ice. After washing twice, samples were run on an LSRFortessa (BD Biosciences) and analyzed with FlowJo software. The following antibodies were used: FITC anti-human CD3, APC anti-human CD69, APC anti-human CD137, APC anti-human CD25, APC anti-human PD1, APC anti-human LAG3, BV421 anti-human CD45RO, APC 
anti-human CD62L, APC anti-human CD3, FITC anti-human CD19, FITC anti-human CD4, APC anti-human CD4, APC anti-human CD8 (All from BioLegend), PerCP-CyTM 5.5 anti-human CD45 (BD Biosciences). For detection of CAR expression, biotinylated human CD19 (aa 20-291) protein (ACRO Biosystems) and PE Streptavidin (BioLegend) were added in order, or PE-labeled human CD19 (aa 20-291) protein (ACRO Biosystems) was used. For some experiments, CAR T cells were co-cultured with target cells at an E/T 1:1 (AAVS1-19bbz experiments) or 1:2 (PD1-19bbz experiments) ratio for 24 hours before harvest. For detection of clinical samples, peripheral blood cells were stained with antibodies, followed by addition of Lysing Buffer (BD Biosciences) before running. CAR percentage was analyzed in $\mathrm{CD} 5^{+} / \mathrm{CD}^{+}$gated cells.

\section{CAR copy number analysis by $q P C R$}

Blood samples were collected before and after CAR T cell infusion. Lysis Buffer (BD Biosciences) was first added and genomic DNA was acquired using Genomic DNA Purification Kit (ThermoFisher). A seven-point standard curve was generated by using $5 \times 10^{0}-5 \times 10^{6}$ copies $/ \mu \mathrm{L}$ lentiviral vector DNA containing the $19 \mathrm{bbz}$ sequence. TaqMan qPCR assay was performed to measure CAR copy number in peripheral blood cells. qPCR was run on a QuantStudio ${ }^{\text {TM }} 3$ Real-Time PCR System (ThermoFisher). Each sample was determined in triplicate. Primers specifically targeting the $19 \mathrm{bbz}$ sequence were as follows: Forward 5'-GCTGTAGCTGCCGATTTCCA-3', Reverse 5'-GGTTCTGGCCCTGCTTGTAC-3', Probe 5'-AGTGAaGTTCAGCAGGAGCGCAGACG-3'.

\section{Antigen stimulation and proliferation of CAR T cells}

As antigen for stimulation, Raji or PD-L1 expressing Raji cells were pre-treated with mitomycin $\mathrm{C}(50 \mu \mathrm{g} / \mathrm{ml})$ for 90 minutes at $37^{\circ} \mathrm{C}$. CAR $\mathrm{T}$ cells were co-cultured with target cells at an E/T 1:1 (AAVS1-19bbz experiments) or 1:2 (PD1-19bbz experiments) ratio for 3-4 days per stimulation. The number of $\mathrm{CAR}^{+}$cells was 
enumerated by multiplying the total cell number and the CAR percentage. Cell viability was measured by Trypan blue staining.

\section{Cell Trace Violet proliferation assay}

AAVS1-19bbz cells were labeled with Cell Trace Violet (ThermoFisher) according to the manufacturer's instructions. Raji cells were pre-treated with mitomycin $\mathrm{C}(50 \mu \mathrm{g} / \mathrm{ml})$ for 90 minutes at $37^{\circ} \mathrm{C}$. CAR $\mathrm{T}$ cells and target cells were mixed at an E/T 1:1 ratio. After 5 days, cells were harvested and run on an LSRFortessa (BD Biosciences).

\section{Bead-based immunoassay}

In preclinical experiments, CAR T cells were co-cultured with Raji or PD-L1 expressing Raji cells at an E/T 1:1 (AAVS1-19bbz experiments) or 1:2 (PD1-19bbz experiments) ratio in media without exogenous cytokines. The supernatant was collected after 24 hours and cytokines were measured using LEGENDplex ${ }^{\text {TM }}$ bead-based immunoassays (BioLegend) according to the manufacturer's instructions.

\section{ELISA}

For in vitro evaluation of infusion products, CAR T cells were co-cultured with Nalm-6 cells at an E/T 1:1 ratio in media without exogenous cytokines. The supernatant was collected after 18-24 hours and IFN- $\gamma$ secretion was measured using Human IFN-gamma ELISA Kit (StemCell) according to the manufacturer's instructions.

\section{Flow cytometry based cytotoxicity assay}

AAVS1-19bbz cells were co-cultured with Raji cells at an E/T 1:1 ratio for 18 hours. Flow cytometry was used to determine residual tumor cells by staining with APC anti-human CD3 and FITC anti-human CD19 antibodies. Cells were enumerated using CountBright ${ }^{\mathrm{TM}}$ Absolute Counting Beads (ThermoFisher) following the manufacturer's instructions. 


\section{LDH cytotoxicity assay}

CAR T cells were co-cultured with Nalm-6, Raji or PD-L1 expressing Raji cells at indicated $\mathrm{E} / \mathrm{T}$ ratios. Cytotoxicity was measured by release of lactate dehydrogenase (LDH) using CytoTox 96® Non-Radioactive Cytotoxicity Assay (Promega) according to the manufacturer's instructions.

648

\section{In vivo mouse experiments}

All animal experiments conformed to the regulations drafted by the Association for Assessment and Accreditation of Laboratory Animal Care in Shanghai and were approved by the East China Normal University Center for Animal Research. For experiments involving AAVS1-19bbz, 6- to 8-week-old NSG male mice were

654

655

656

657

658

659

660

661

662

663

664

665

666

667

668

669

670

671 injected intravenously with $2 \times 10^{5}$ ffLuc-transduced Raji cells. $2 \times 10^{6} \mathrm{CAR}$ T cells were administered intravenously after 5 days. For experiments using PD1-19bbz, 6to 8-week-old NSG male mice were inoculated intravenously with $5 \times 10^{5}$ ffLuc-transduced PD-L1 expressing Raji cells. $5 \times 10^{6}$ CAR T cells were injected intravenously after 10 days. Bioluminescence images were acquired and analyzed using IVIS Imaging System and software (PerkinElmer).

\section{Statistics}

Experimental data are presented as mean \pm SD or mean \pm SEM as described in the figure legends. Data were analyzed by one-way ANOVA or two-way ANOVA as indicated using GraphPad software. A p value $<0.05$ was considered statistically significant. Asterisks used to indicate significance correspond to ***p $<0.001, * * \mathrm{p}<0.01,{ }^{*} \mathrm{p}<0.05$. NS, nonsignificance.

\section{Acknowledgements}

We thank Stefan Siwko for discussing and revising this manuscript. This study was supported by National Key R\&D Program of China (2019YFA0802802, 
672 2018YFA0507001), National Natural Science Foundation of China (81772622, 673 91857116, 31871453, 81730008, 81770201), Innovation Program of Shanghai 674 Municipal Education Commission (2017-01-07-00-05-E00011), Key Project of 675 Science and Technology Department of Zhejiang Province (2019C03016) and Natural 676 Science Foundation of Shanghai (18ZR1412300).

677

678 Author contributions

679 J.Z., Y.H., B.D., M.L. and H.H. designed the overall study and wrote the manuscript. 680 Y.H., W.L. and H.H. designed the clinical trial. J.Z., J.Y., Y.T., L.Z. and Y.Q. 681 performed the experiments. J.Z., B.T., Q.T. and Q.W. were responsible for 682 manufacturing and quality control of CAR T cells. Y.H., M.Z., G.W., K.Z., J.C. and 683 Y.L. performed the clinical trial. J.Z., Y.H. and W.L. analyzed the data. Y.W. and D.L. 684 discussed the results and manuscript. B.D., M.L. and H.H. supervised the study. All 685 authors approved the article for submission and publication.

686

687

\section{Competing interests}

688 This study was partially supported by Bioray Laboratories Inc. Patents related to this 689 manuscript have been applied.

690

691

692

693

694

695

696

697

698

699

700

701

702

703

704

705

706

707 

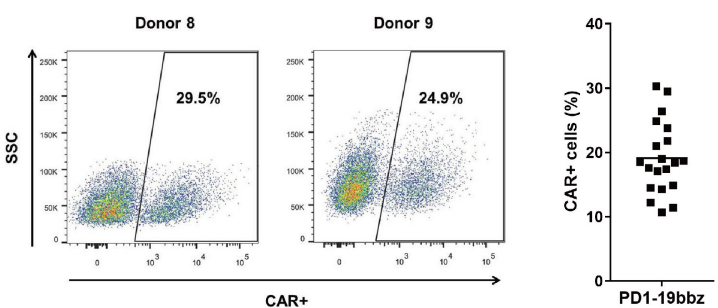

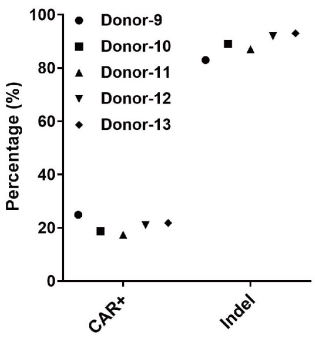

h

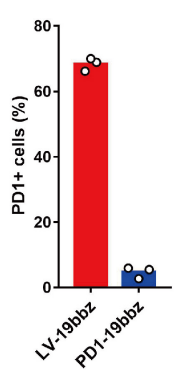

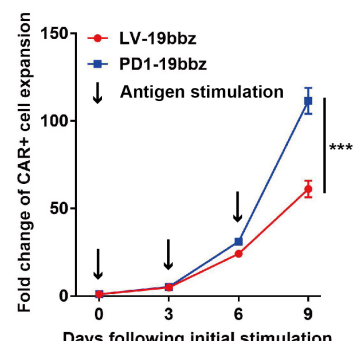

Days following initial stimulation

j

CD69

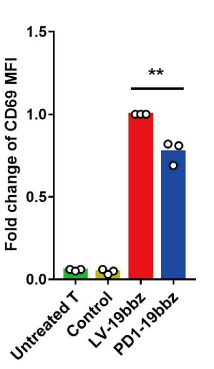

CD137

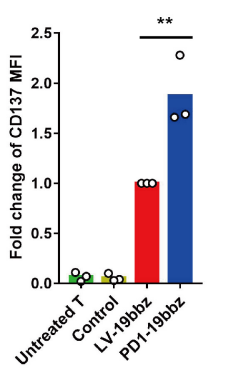

CD25

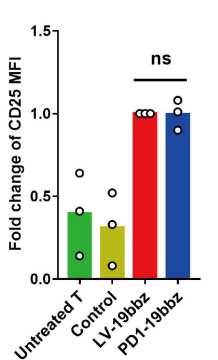

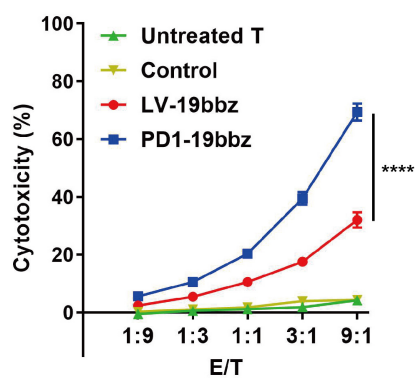

i
IL-2

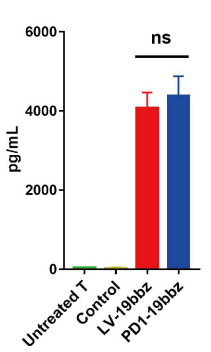

TNF- $\alpha$

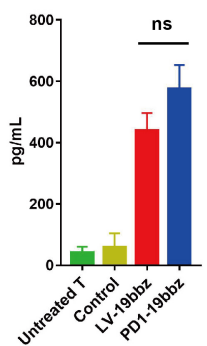

IFN-Y

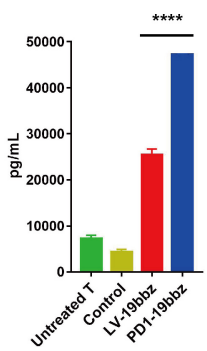

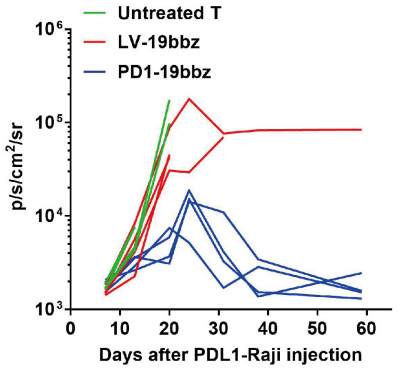

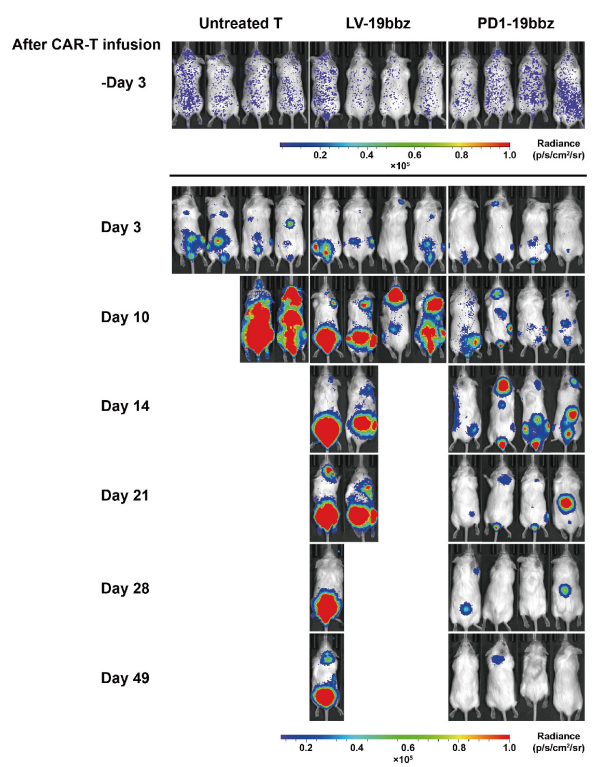

Figure 2 Non-viral PD1-integrated CAR T cells outperform conventional CAR T cells

a, CAR expression determined 7 days after electroporation of cells from two representative healthy donors. $b$, Percentage of CAR+ cells detected 7 days after electroporation ( $n=20$ independent healthy donors). c, Percentages of CAR integration and PD1 indels in total T cells were detected 7 days after electroporation in five representative healthy donors. $d$, Percentage of PD1 expression detected by flow cytometry in CD3+/CAR+ gated cells after 24 hours co-culture with PD-L1 expressing Raji cells ( $n=3$ independent healthy donors). e, Expansion of CAR+ cells after repeated stimulation with PD-L1 expressing Raji cells. Data are mean \pm SD ( $n=3$ technical replicates). f, MFI of CD69, CD137 and CD25 expression in T cells detected by flow cytometry after 24 hours co-culture with PD-L1 expressing Raji cells ( $n=3$ independent healthy donors). CD3+ (Untreated T, Control) or CD3+/CAR+ (LV-19bbz, PD1-19bbz) gated cells were analyzed. g, Representative result of cytokine secretion in the supernatant (measured by bead-based immunoassay) after co-culture with PD-L1 expressing Raji cells for 24 hours. Data are mean \pm SD ( $=2$ technical replicates). h, In vitro cytotoxicity against PD-L1 expressing Raji cells determined by LDH assay. Data are mean \pm SD ( $n=3$ technical replicates). i-j, Bioluminescence kinetics (i) and imaging (j) of PD-L1 expressing Raji tumor cell growth in NSG mice following different treatments $(n=4)$. Control samples were electroporated the same as PD1-19bbz cells except without sgRNA addition. Mean value is shown in $b$, d, f. P values are calculated by one-way ANOVA (f, g) or two-way ANOVA (e, h). 

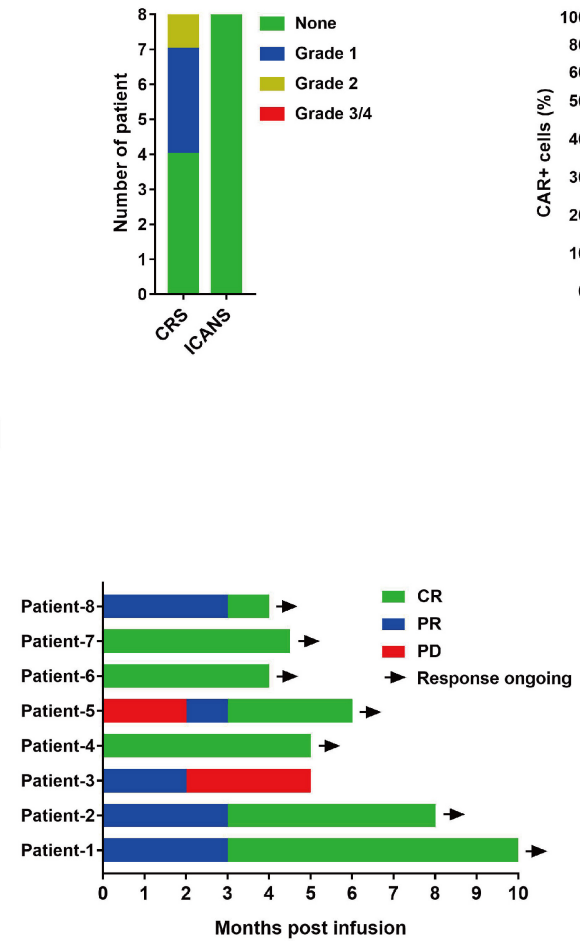
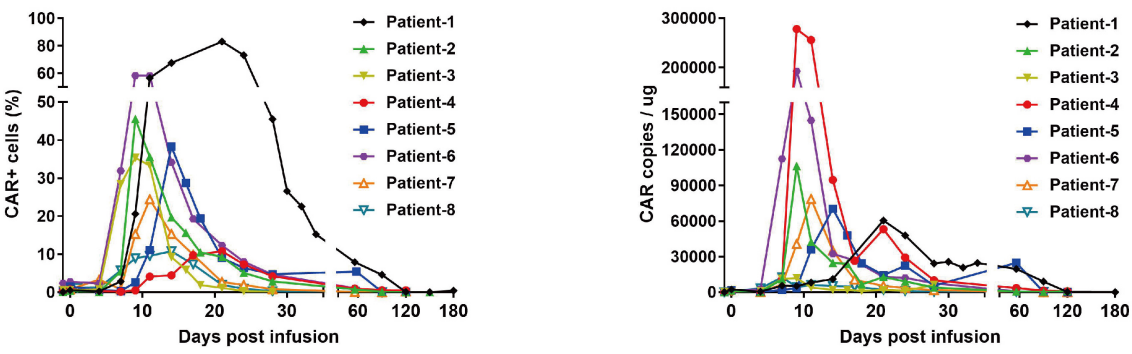

e
Patient-2 (Before treatment)

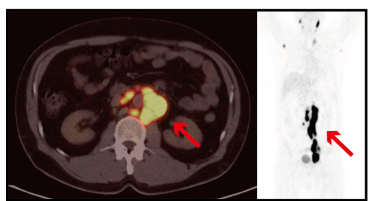

Patient-2 (90 days after treatment)

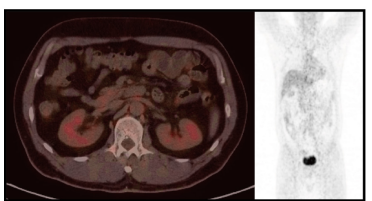

Patient-4 (Before treatment)

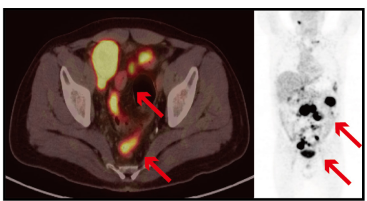

Patient-4 (28 days after treatment)

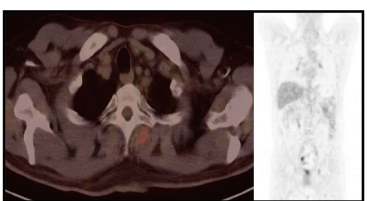

Patient-6 (Before treatment)

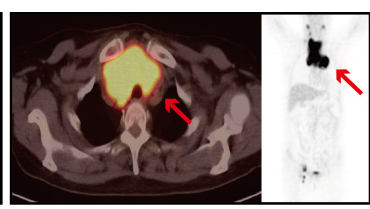

Patient-6 (28 days after treatment)

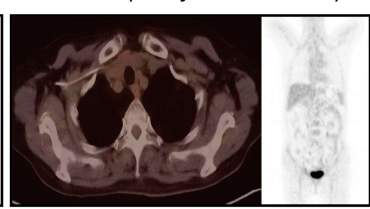

Figure 3 Non-viral PD1-integrated CAR T cells potently eliminate tumor cells in patients with r/r B-NHL without serious toxicity

a, Percentages of CRS and ICANS occurrence after treatment. CRS, cytokine release syndrome. ICANS, immune effector cell-associated neurotoxicity syndrome. $b$, Percentage of CAR+ cells in the peripheral blood T cells of patients on indicated days before and after infusion. c, CAR copy number in genomic DNA from the peripheral blood of patients is shown on indicated days before and after infusion. $d$, Treatment responses and duration of responses after infusion. CR, complete remission. PR, partial remission. PD, progressive disease. e, PET-CT scans for three representative patients before and after treatment. Red arrows indicate the tumor lesions. 
a

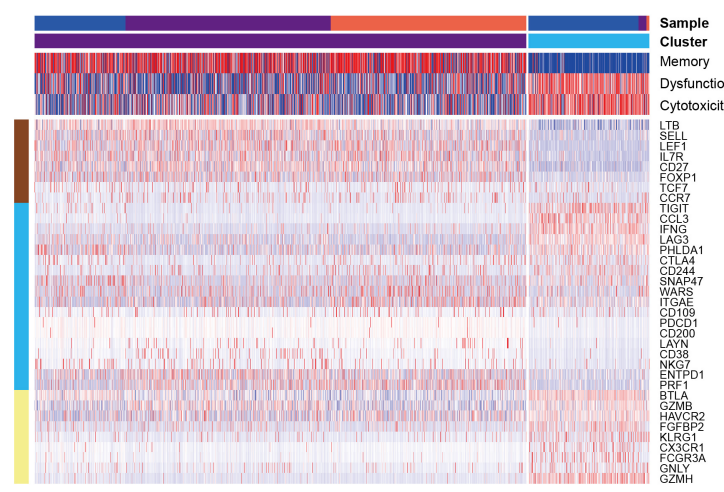

C
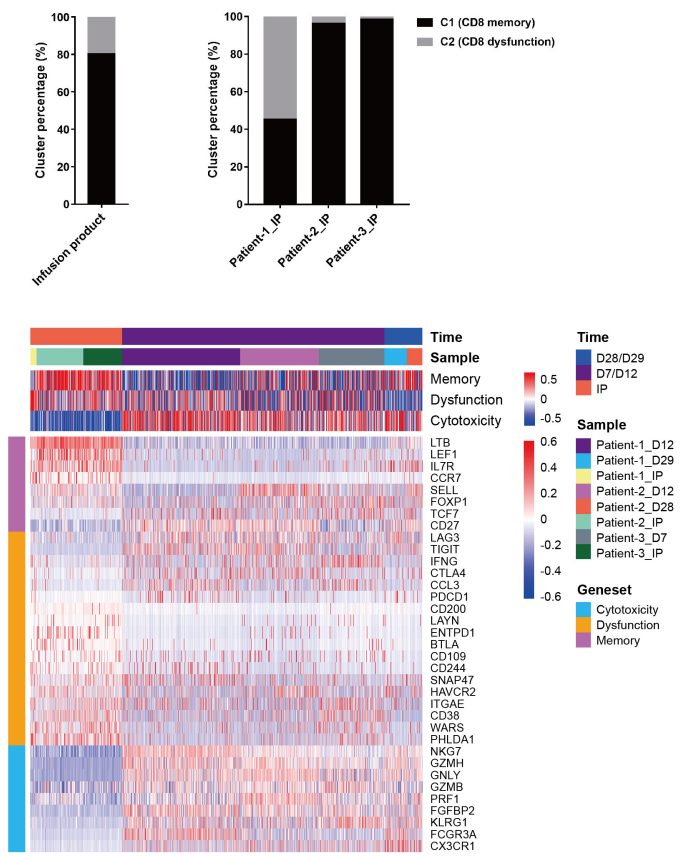

b d

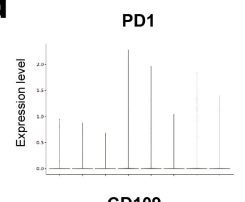

CD109

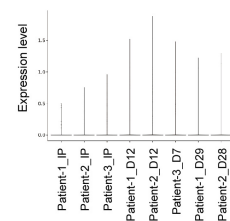

ENTPD1

CXCL13

BTLA

CD244

CD200
Sample

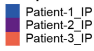

Cluster $\mathrm{C} 1$
$\mathrm{C} 2$ Geneset
Cytotoxicity
Dysfunction e

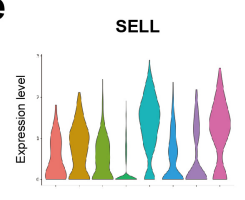

TCF7

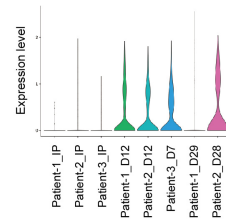

f

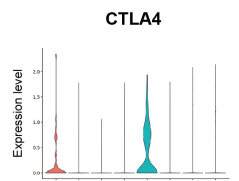

CCL3

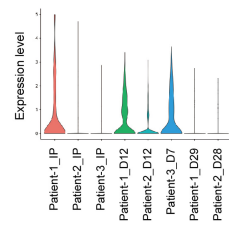

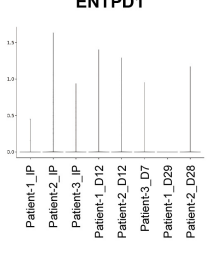

IL7R

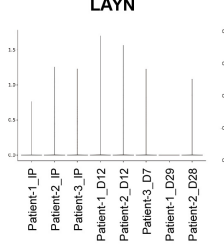

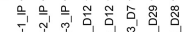
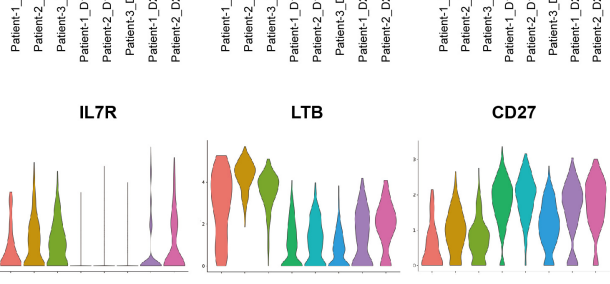

FOXP1

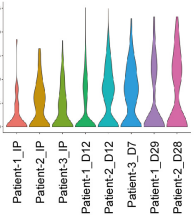

ITGAE

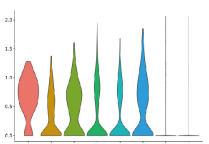

SNAP47

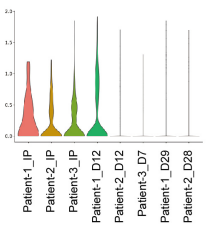

IFNG

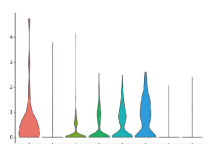

PHLDA1

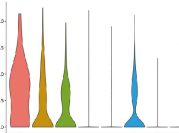

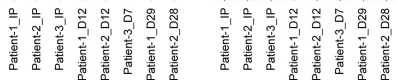

\section{Figure 4 Single-cell RNA sequencing of non-viral PD1-integrated CAR T cells before and after infusion}

a, Heat map showing scaled expression of memory, dysfunction and cytotoxicity genes in two CD8+ T cell clusters in three infusion products (IP). The scGSVA scores of CD8 memory, dysfunction and cytotoxicity signatures are shown at the top. Cluster 1 (C1) and cluster 2 (C2) were generated by clustering CD8 memory and dysfunction marker genes, respectively. $b$, Percentages of $C 1$ and $\mathrm{C} 2$ in mixed and individual samples of infusion products. c, Heat map showing scaled expression of memory, dysfunction and cytotoxicity genes in CD8+/CAR+ cells from three patients before and after infusion. The scGSVA scores of CD8 memory, dysfunction and cytotoxicity signatures are shown at the top. d-f, Violin plots showing the expression of PD1 (d), PD1-associated inhibitory genes (d), memory genes (e) and dysfunction genes (f) in CD8+/CAR+ cells from three patients before and after infusion. The data of patient-3 sample after 28 days treatment is not shown due to an unreliable low CAR+ cell number. 
C

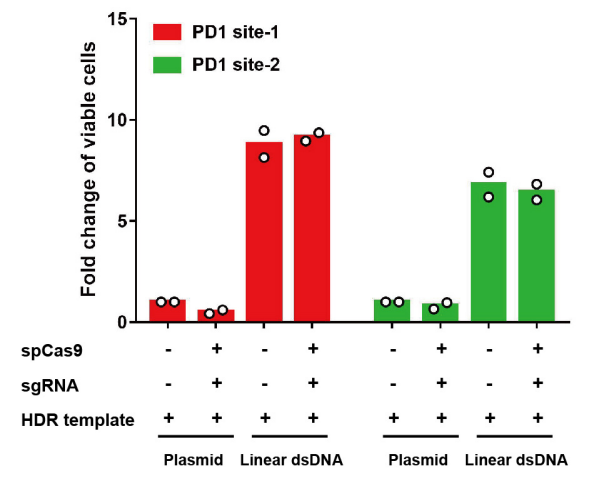

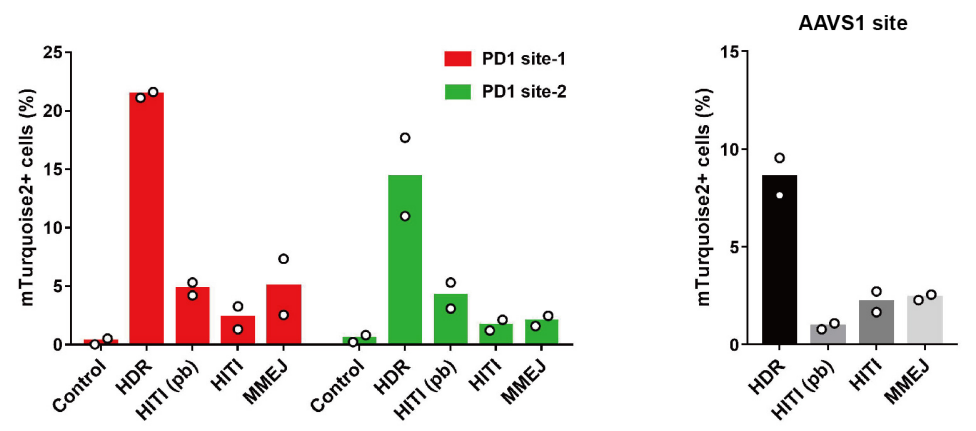

d

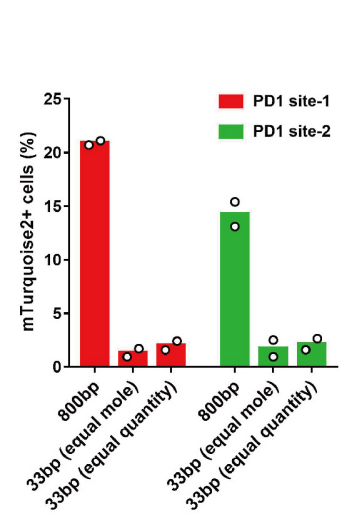

g e

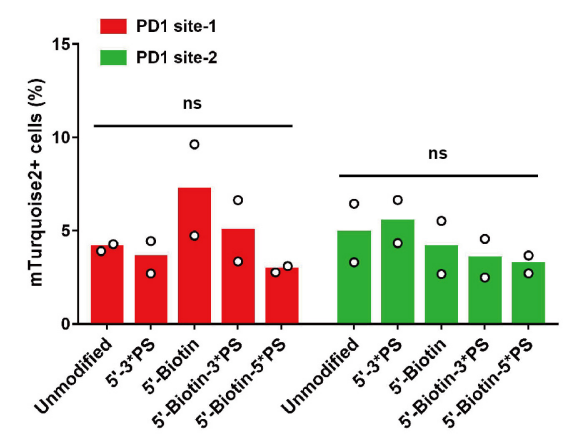

PD1 site-1

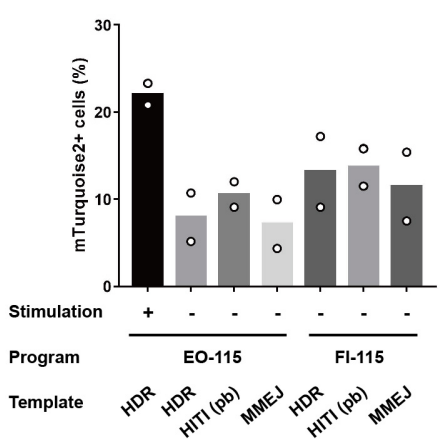

PD1 site-1

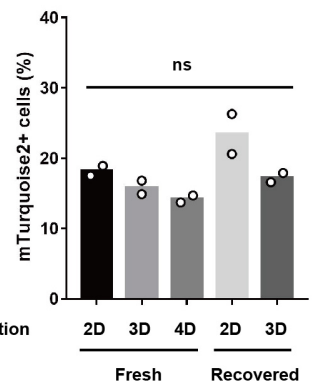

Supplementary figure 1 Optimization of the conditions for constructing non-viral genome specific targeted T cells

The sequence of fluorescent protein mTurquoise 2 was used as a target to optimize the conditions for generating non-viral genome specific integrated T cells. a, Number of viable cells calculated 7 days after electroporation by using different protocols. Equal quantities of circular plasmid DNA and linear double-stranded DNA (dsDNA) were used. Due to acquisition of higher cell viability, templates in the form of linear dsDNA were chosen for all the following experiments. b-c, Recombination efficiency of mTurquoise2 at two PD1 sites (b) and one AAVS1 site (c) by using different DNA templates. HDR, homology directed repair. HITI, homology-independent targeted integration. HITI (pb), HITI template with 50bp protection base pairs flanking the target sequence. MMEJ, microhomology-mediated end joining. $d$, Recombination efficiency of mTurquoise 2 using $33 \mathrm{bp}$ or $800 \mathrm{bp}$ homology arms. Equal mole or quantity of template harboring $33 \mathrm{bp}$ homology arms was used, compared with template with 800 bp homology arms. e, Recombination efficiency of mTurquoise 2 by using unmodified or modified DNA templates with $200 \mathrm{bp}$ homology arms. PS, phosphorothioate. Biotin was modified at the first base pair from the 5 ' side. PS was modified at the first three or five base pairs from the 5 ' side. f, Recombination efficiency of $m$ Turquoise 2 in unstimulated or stimulated T cells using different programs and DNA templates. $g$, Recombination efficiency of mTurquoise 2 in fresh or recovered T cells after stimulation for indicated days by using HDR templates with $800 \mathrm{bp}$ homology arms. $800 \mathrm{bp}$ and $20 \mathrm{bp}$ homology arms were used in HDR and MMEJ templates, respectively. Equal moles of DNA template were used in b, c, e-g. The recombination efficiency was determined 7 days after electroporation in $\mathrm{b}-\mathrm{g}$. All the experiments were performed in cells from two independent healthy donors. Mean value is shown in all the figures. $P$ values are calculated by one-way ANOVA $(g)$ or two-way ANOVA (e). 

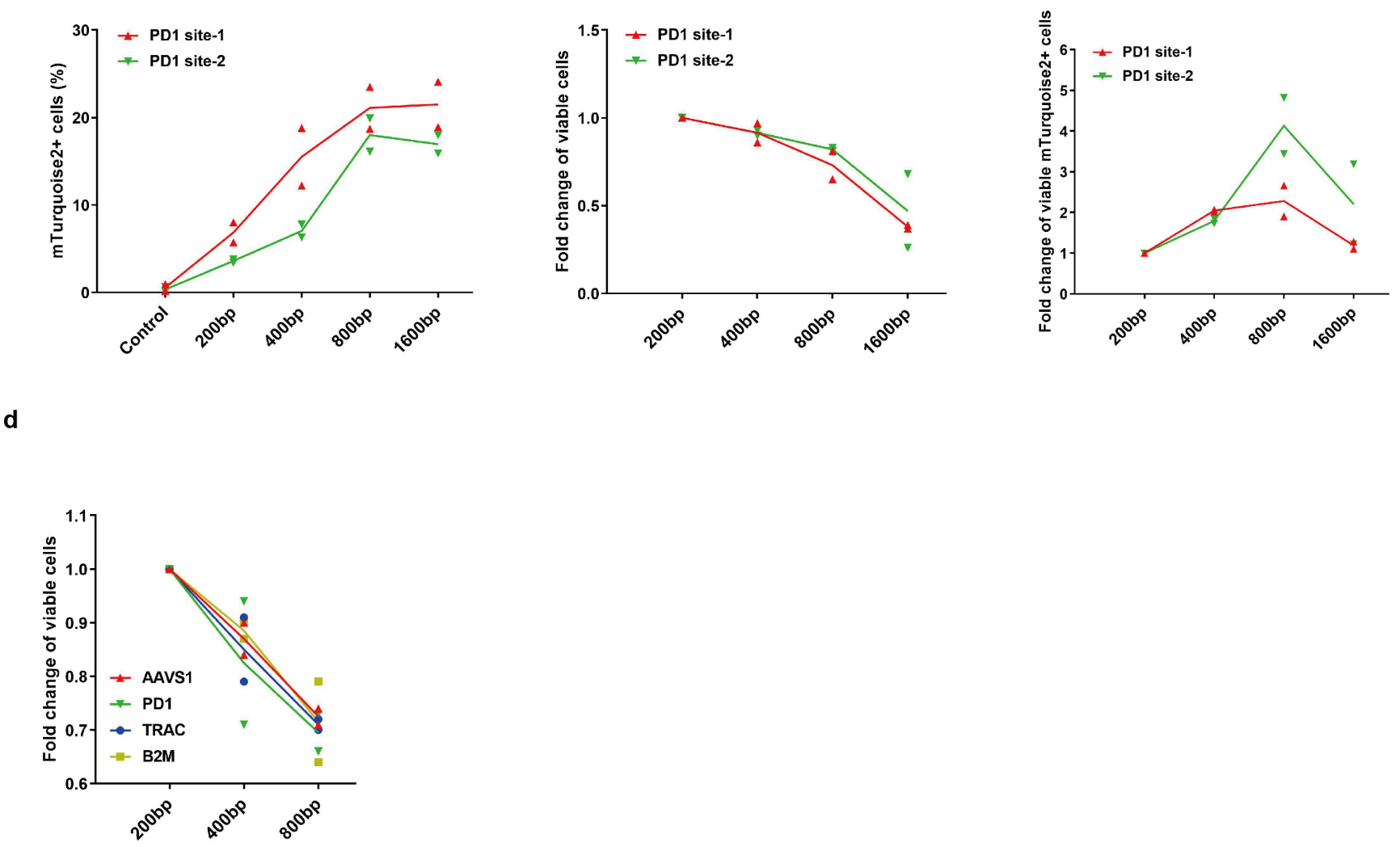

Supplementary figure 2 Comparison of recombination efficiency and cell viability among templates with different length of homology arms

The sequence of fluorescent protein mTurquoise 2 was used as a target to compare different homology arm lengths in a-c. a-c, Recombination efficiency (a) and numbers of all viable cells (b) and viable mTurquoise2+ cells (c) were detected 7 days after electroporation using equal molar mTurquoise2 templates with different homology arm lengths. $d$, Number of all viable cells was enumerated 7 days after electroporation using equal molar CAR templates with different homology arm lengths. All the experiments were performed in two independent healthy donors. Mean value is shown in all the figures.

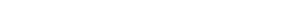




\section{Supplementary figure 3}

\section{b}

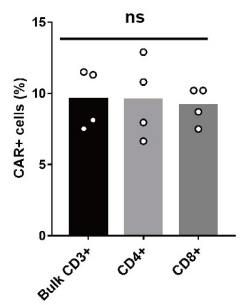

f
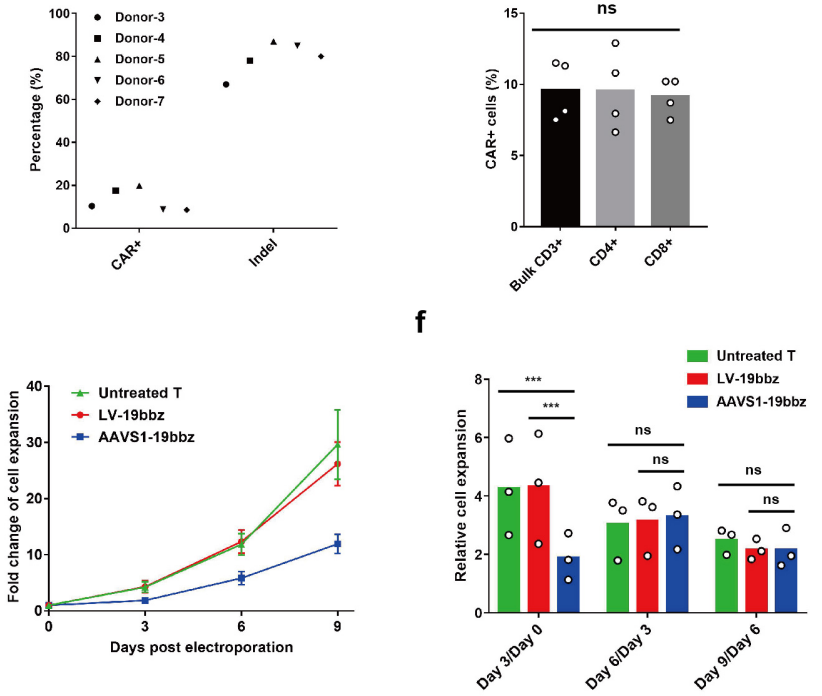

C

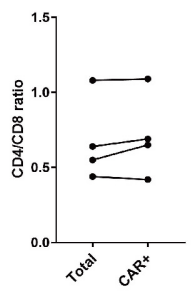

g

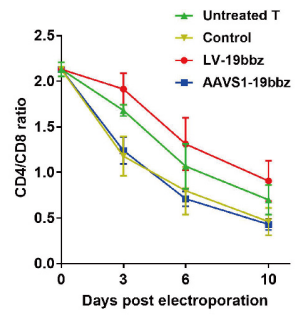

d

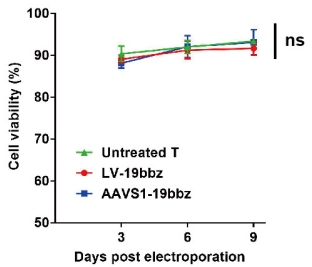

h

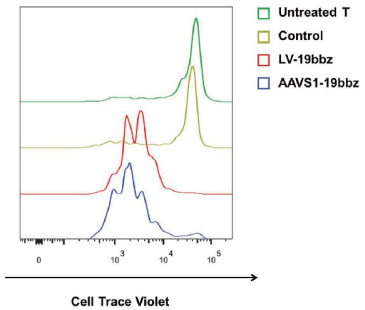

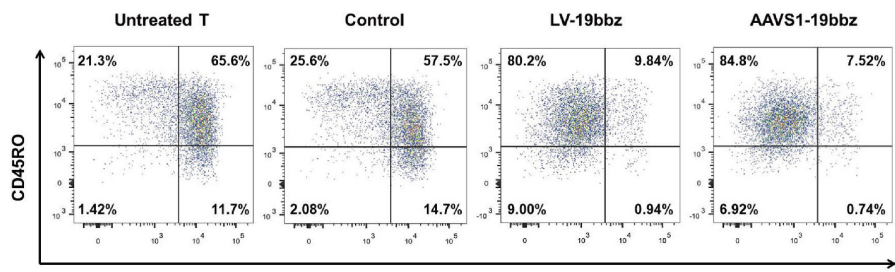

CD62L

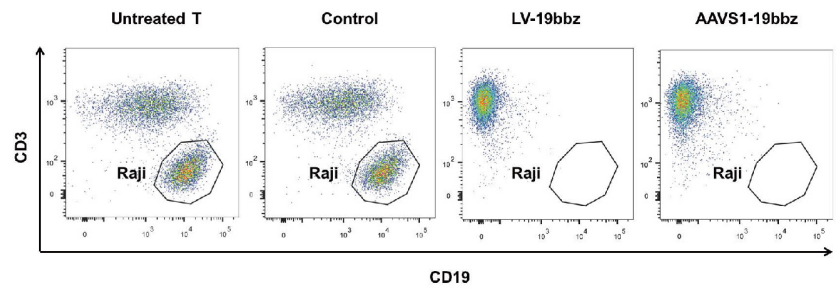

k

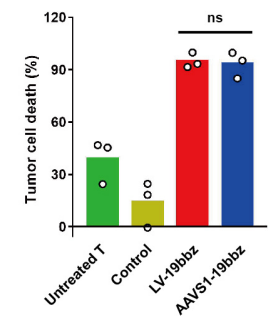

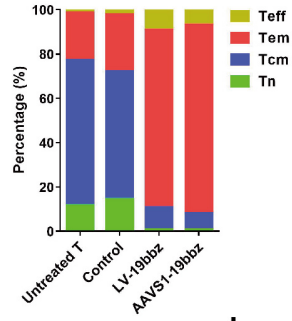

I

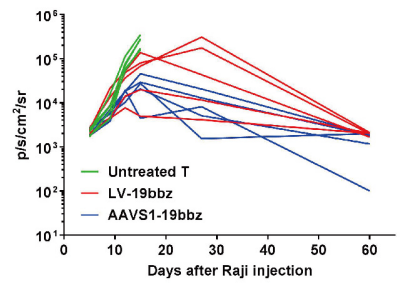

Supplementary figure 3 Non-viral AAVS1-integrated CAR T cells behave comparably to conventional CAR T cells

a, Percentages of CAR integration and AAVS1 indels in total T cells were detected 7 days after electroporation in five representative healthy donors. b, Percentages of CAR integration in CD3+, CD4+ and CD8+ cells determined 7 days after electroporation $(n=4$ independent healthy donors). c, Comparison of CD4/CD8 ratio between total and CAR+ cells ( $n=4$ independent healthy donors). $d$, Cell viability detected by trypan blue staining on indicated days post electroporation. Data are mean \pm SEM ( $n=3$ independent healthy donors). e-f, Absolute (e) and relative (f) rates of $T$ cell growth in vitro ( $n=3$ independent healthy donors). Data are mean \pm SEM in e. g, Ratio of CD4+ and CD8+ cells on indicated days post electroporation. Data are mean \pm SEM ( $n=3$ independent healthy donors). h, Representative histogram showing Cell Trace Violet staining of T cells after co-culture with mitomycin C-treated Raji cells for 5 days. i, Representative flow cytometry plots showing CD45RO/CD62L expression in T cells after 24 hours co-culture with Raji cells. The T cell subset differentiation is shown at right. j, Representative flow cytometry plots showing lysis of Raji cells following 18 hours co-culture. $k$, The percentage of Raji tumor cell death detected by flow cytometry-based cytotoxicity assay ( $\mathrm{n}=3$ independent healthy donors). I, Bioluminescence kinetics of Raji tumor cell growth in NSG mice following different treatments $(n=5)$. Control samples were electroporated the same as AAVS1-19bbz cells except without sgRNA addition. CD3+ (Untreated T, Control) or CD3+/CAR+ (LV-19bbz, AAVS1-19bbz) gated cells are analyzed in $h$, i. Mean value is shown in $b, f, k$. P values are calculated by one-way ANOVA (b, $k$ ) or two-way ANOVA (d, f). 


\section{Supplementary figure 4}

\section{a}

Sanger sequencing

\begin{tabular}{|c|c|c|c|}
\hline \multirow{3}{*}{ Edited locus } & \multicolumn{3}{|c|}{ ler } \\
\hline & & $19 \mathrm{bbz}$ & \\
\hline & 5' homology arm & $\longleftarrow$ & 3' homology arm \\
\hline & & $\begin{array}{l}\text { AAVS1-19bbz } \\
\text { PD1-19bbz }\end{array}$ & \\
\hline
\end{tabular}
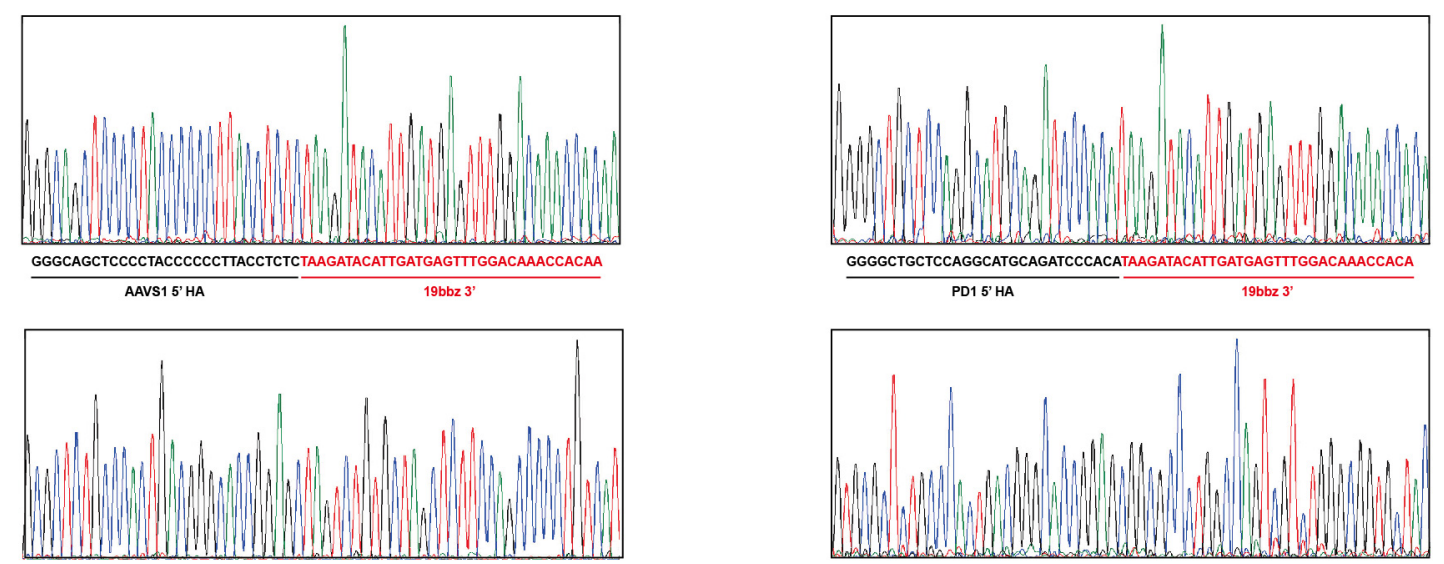

GTGCGCTCTGCCCACTGACGGGCACCGGAGCGGCGCCCTGGCCAGTCGTCTGGGCGGTGCTAC

$\frac{\text { GCGCTCTGCCCACTGACGGGCACCGGAGCTAGTCTGTGCTAGCTCTTCCAGCCCCCTGTCAT }}{19 \mathrm{bbz} 5^{\prime}}$ $19 \mathrm{bbz} 5^{\prime}$ PD1 3' HA

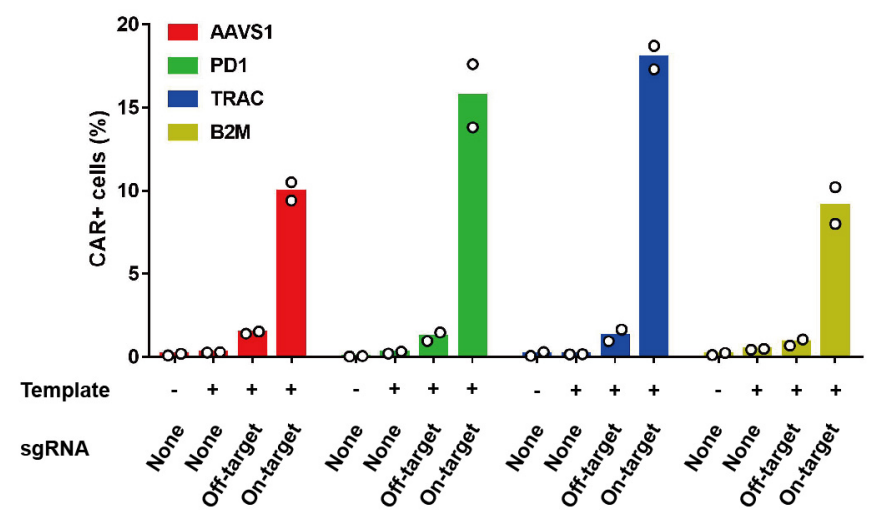

Supplementary figure 4 Site-specific integration of CAR cassette

a, For the samples of AAVS1-19bbz and PD1-19bbz, CAR+ cells were sorted by fluorescence-activated cell sorting (FACS). Genomic DNA was used as template to amplify PCR products across the homology arms. Sanger sequencing was performed from end to end, outside of homology arms. b-c, Sequences of $5^{\prime}$ and $3^{\prime}$ 'junction sites between the homology arm and CAR cassette at the AAVS1 (b) and PD1 (c) locus. d, Non-specific integration of CAR elements was tested 7 days after electroporation by using different combinations of DNA template and sgRNA ( $\mathrm{n}=2$ independent healthy donors). For the groups of AAVS1, PD1 and TRAC templates, one B2M sgRNA with high cleavage efficiency was used as off-target sgRNA. For the B2M template group, one TRAC sgRNA with high cleavage efficiency was used as off-target sgRNA. The off-target groups were designed to detect non-targeted integration under a hypothesized condition that sgRNA had very high off-target cleavage efficiency. Mean value is shown in d. 
Supplementary figure 5

a

b

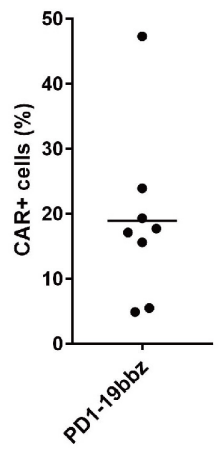

d

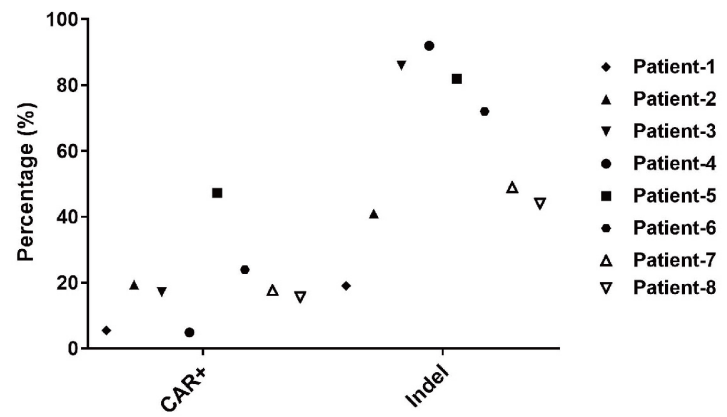

e

Patient-6
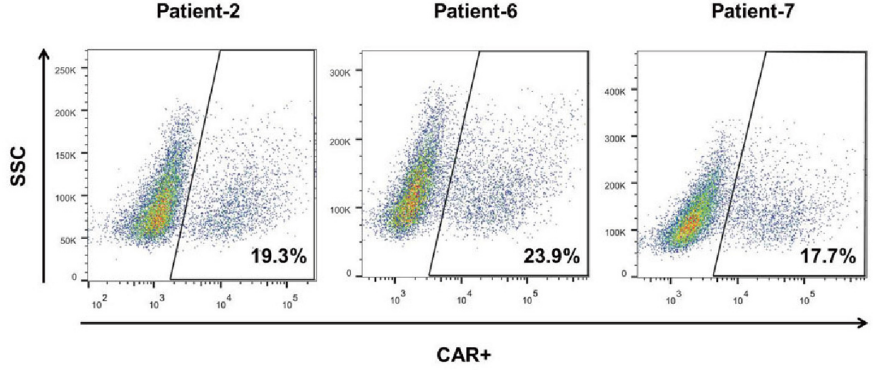

CAR+

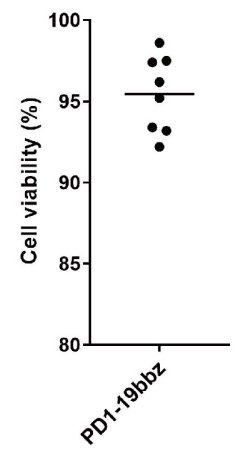

C

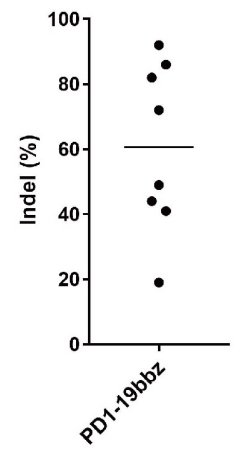

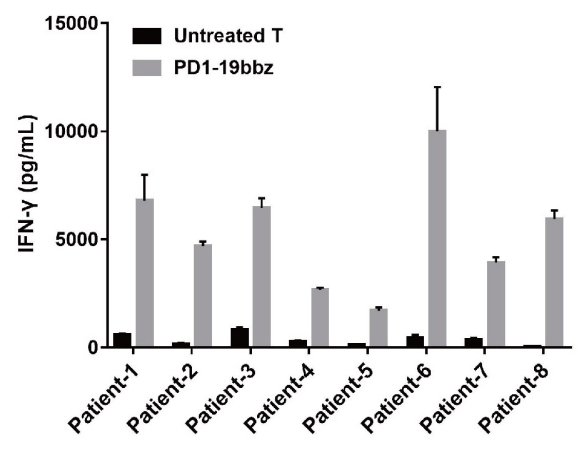

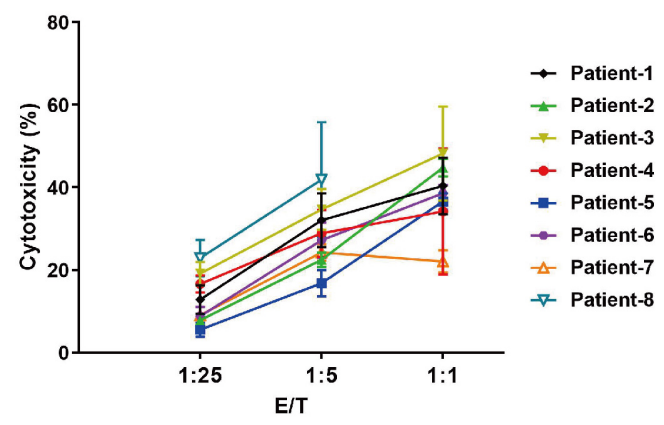

Supplementary figure 5 In vitro evaluation of non-viral PD1-targeted CAR T cell products

a, Percentage of CAR+ cells in the final products of eight r/r B-NHL patients. b, CAR expression determined in three representative patient donors. c-d, Percentages of CAR integration (d) and $P D 1$ indels $(c, d)$ in the final products. e, Cell viability of the final products detected by trypan blue staining. $f$, IFN- $\gamma$ secretion measured by ELISA in the supernatant after co-culture with Nalm- 6 cells for 18-24 hours. Data are mean $\pm \mathrm{SD}$ ( $n=3$ technical replicates). $\mathrm{g}$, In vitro cytotoxicity against Nalm-6 cells determined using LDH assay. E/T, effector/target. Data are mean $\pm S D$ ( $n=3$ technical replicates). Mean value is shown in a, c, e. 


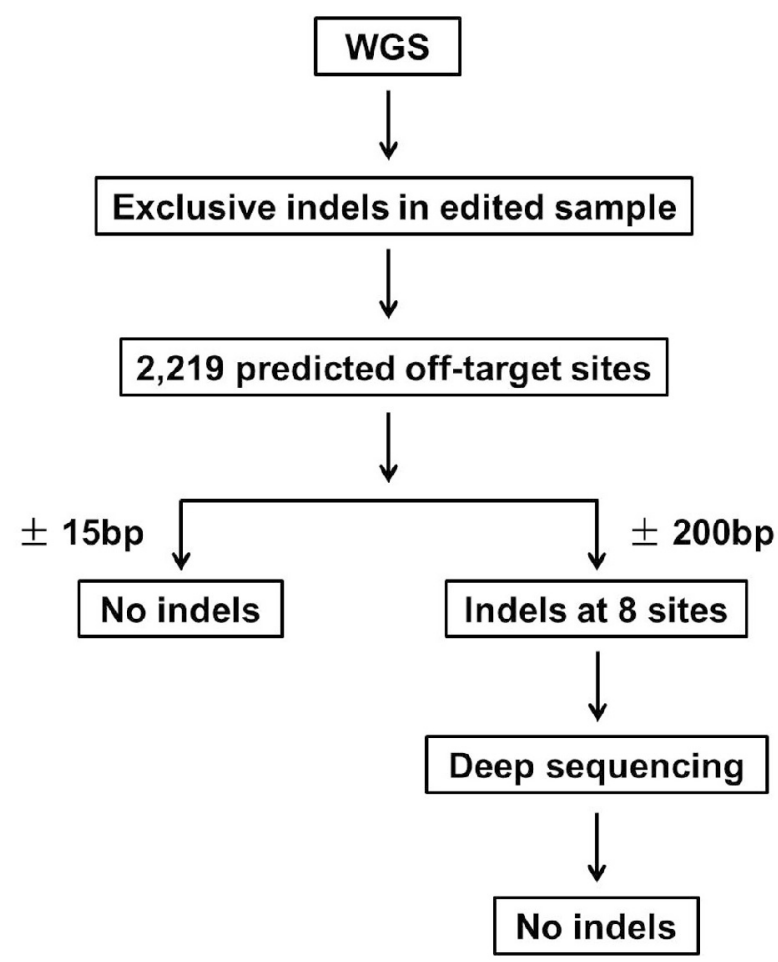
around the on-target site) were predicted by Cas-OFFinder and compared with exclusive indels in the edited sample by bioinformatics. No indel events were detected within $15 \mathrm{bp}$ upstream and downstream $( \pm 15 \mathrm{bp})$ of the sites. Indels were found within $200 \mathrm{bp}$ upstream and downstream $( \pm 200 \mathrm{bp})$ of eight sites. Deep sequencing was then performed to validate these indel events. While no indels were detected at five sites, indels at the other three sites were variances of one unit length on nucleotide repeats and thus were not considered to be true off-target events. 
Patient-1

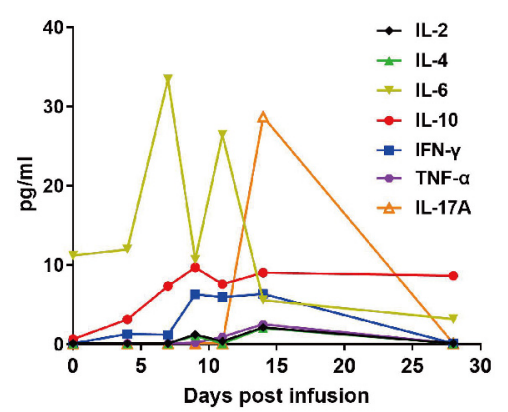

Patient-4

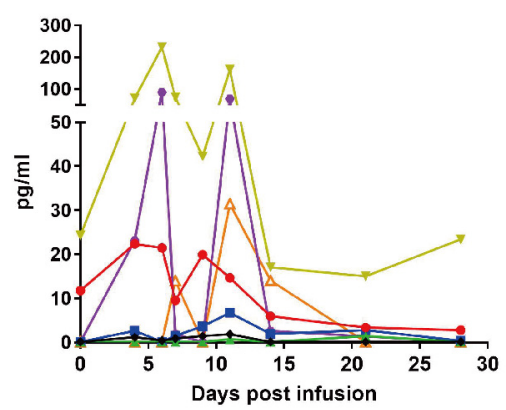

Patient-7

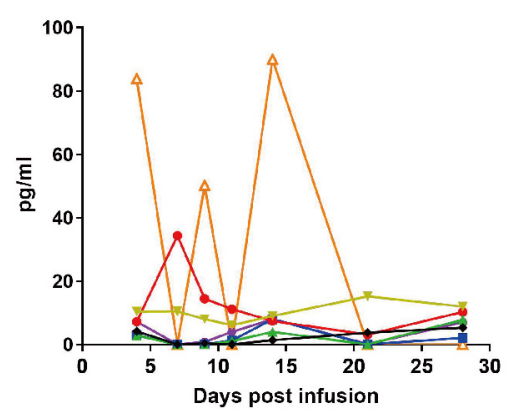

Patient-2

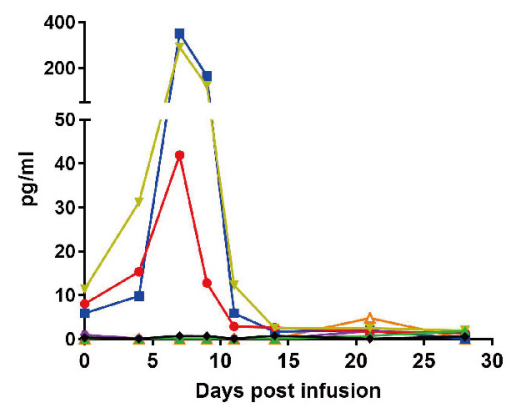

e

Patient-5

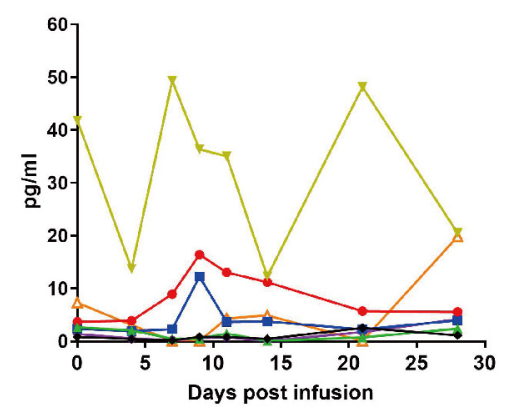

h

Patient-8

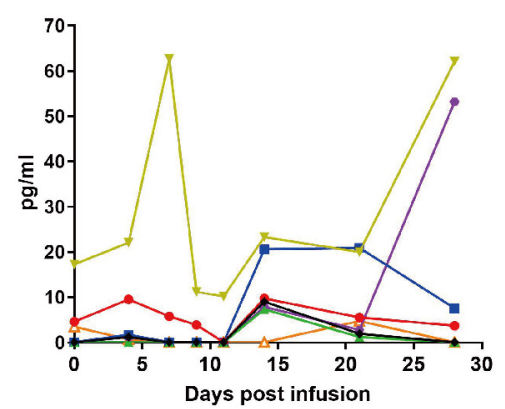

Patient-3

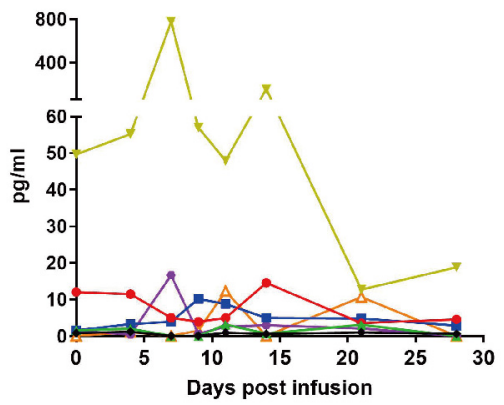

f

Patient-6

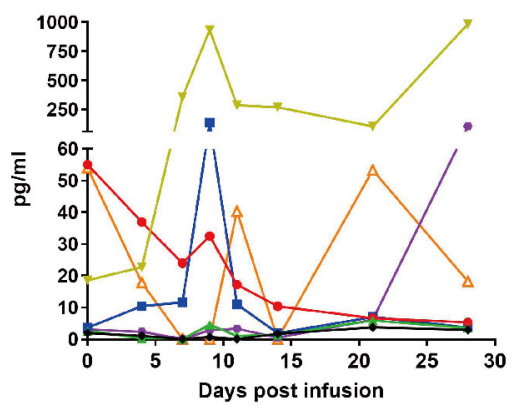

Supplementary figure 7 Serum cytokine profiles in r/r B-NHL patients after treatment of non-viral PD1-targeted CAR T cells $\mathrm{a}-\mathrm{h}$, Serum cytokines including IL-2, IL-4, IL-6, IL-10, IFN- $\gamma$, TNF- $\mathrm{a}$ and IL-17A were assessed in eight $\mathrm{r} / \mathrm{r}$ B-NHL patients on indicated days after infusion. 


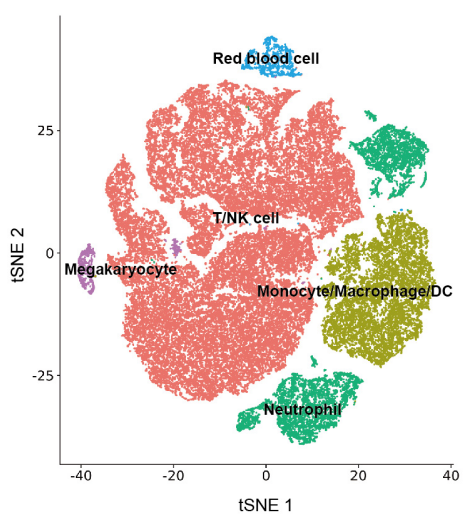
d

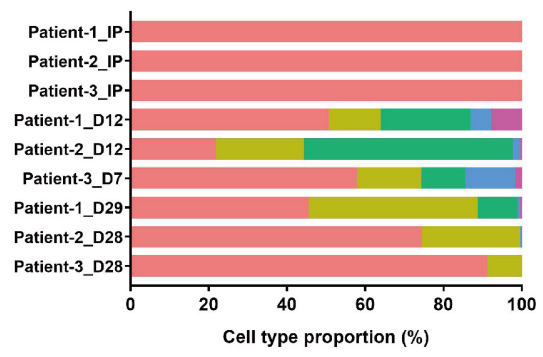

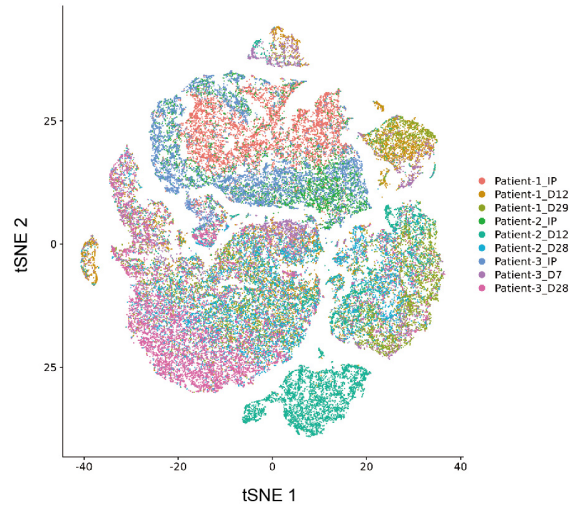

e
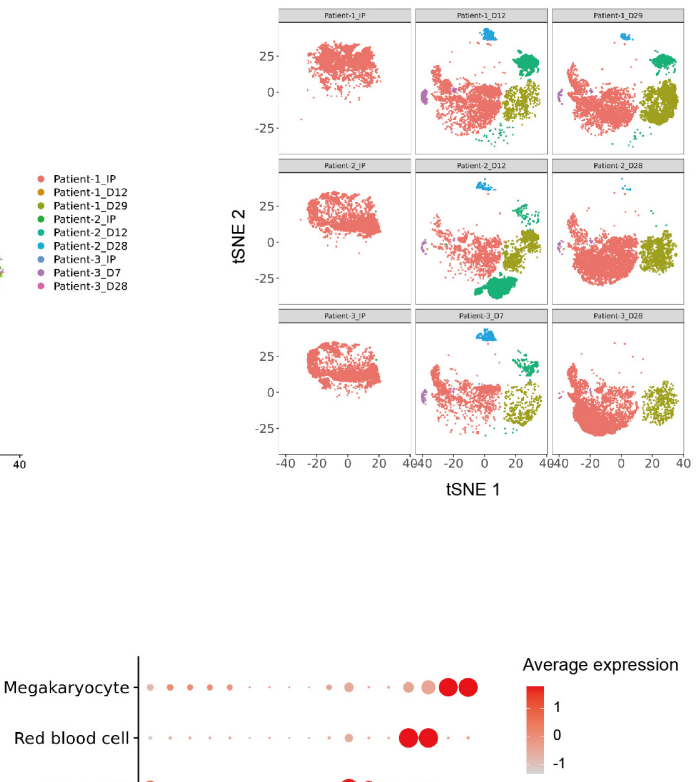

Percent expressed

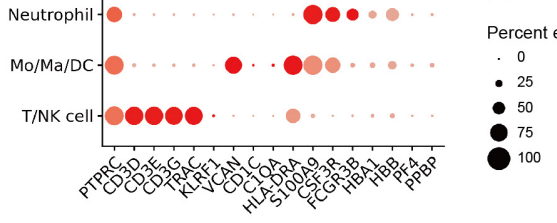

\section{Supplementary figure 8 Overview of the single-cell landscape}

a-b, Overview of the 54,774 cells that passed QC for single-cell analysis. Cells are color coded by cell type (a) and patient sample (b), respectively, in t-distributed stochastic neighbor embedding (tSNE) plots. c, tSNE plot showing cell clusters in each sample. d, Proportion of cell types in each patient sample. e, Bubble heat map showing marker gene expression for different cell types. 


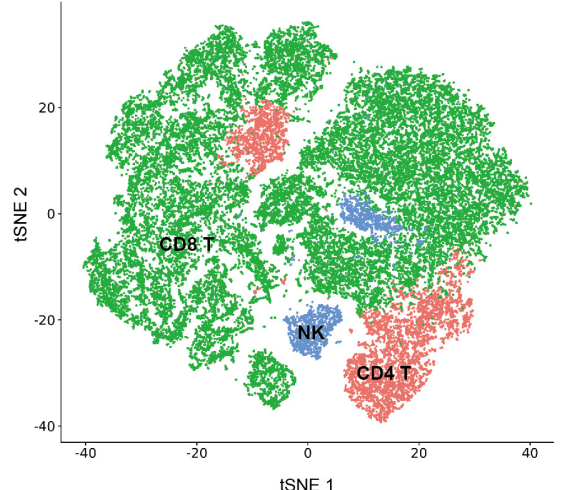

C

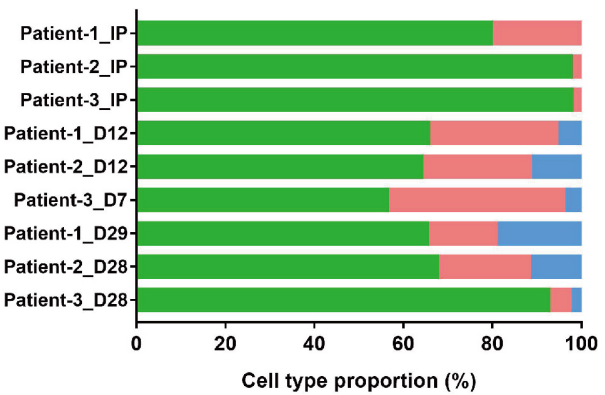

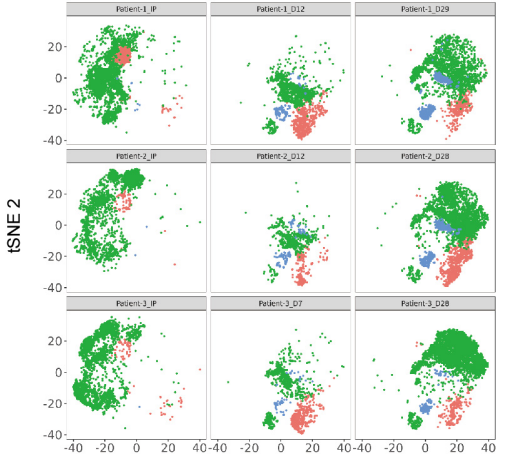

tSNE 1

d

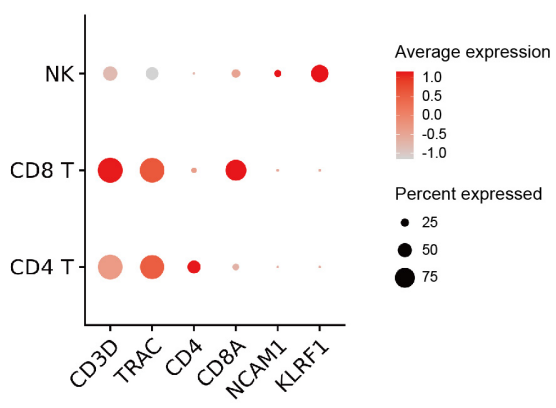

\section{Supplementary figure 9 Landscape of T/NK cell types in single-cell analysis}

a, Overview of the 36,201 cells in the T/NK cell cluster. Cells are color coded by cell type in the tSNE plot. b, tSNE plot showing subtypes in the T/NK cell cluster in each patient sample. c, Proportion of subtypes in the T/NK cell cluster in each sample. d, Bubble heat map showing marker gene expression for different subtypes in the T/NK cell cluster. 
a

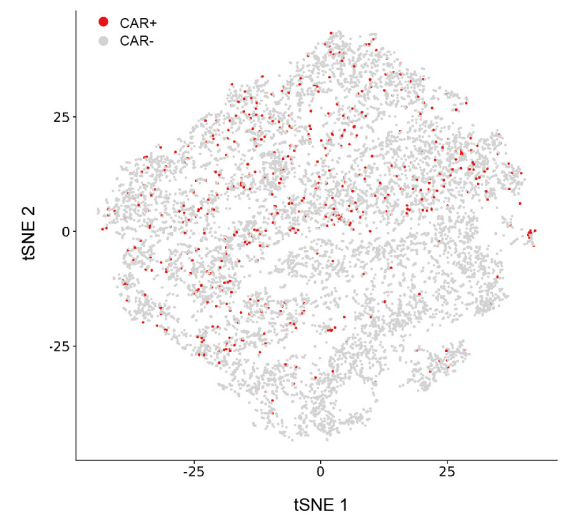

e

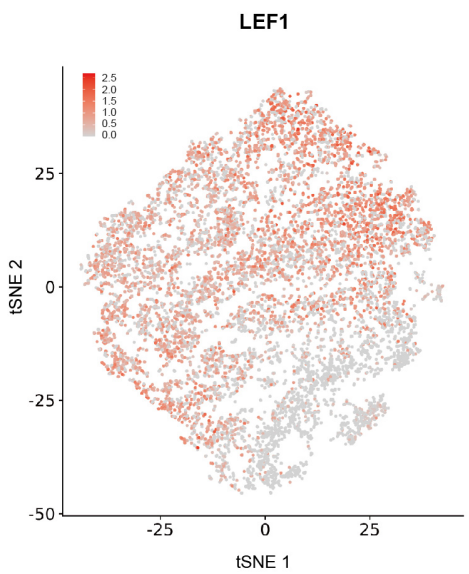

h

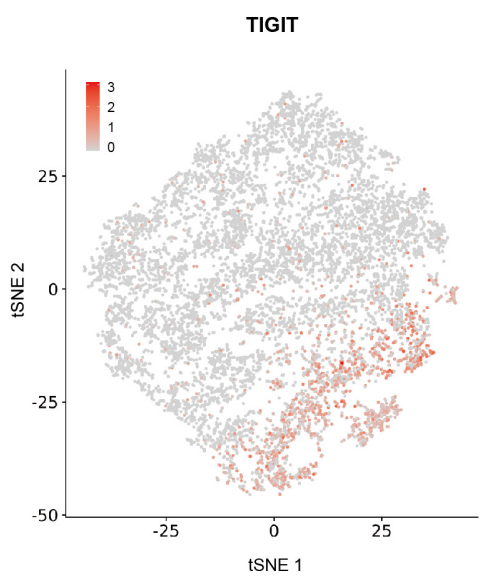

b

c

SELL
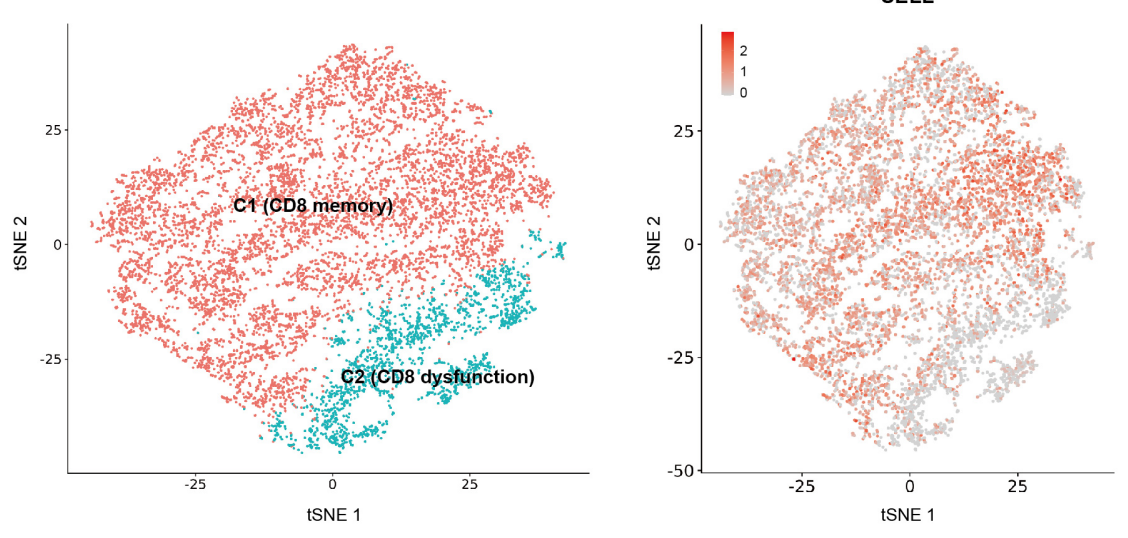

f

IL7R
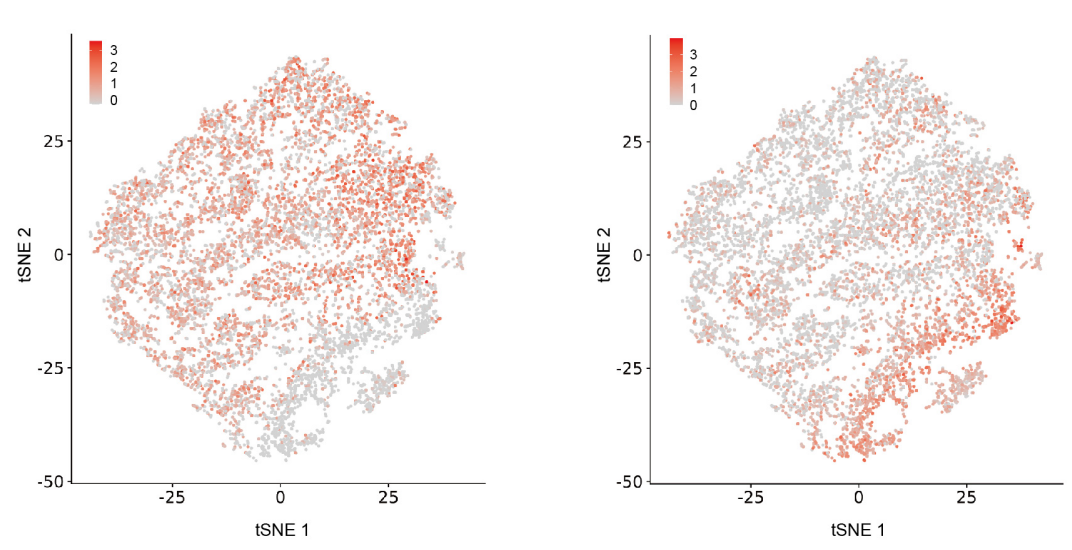

i

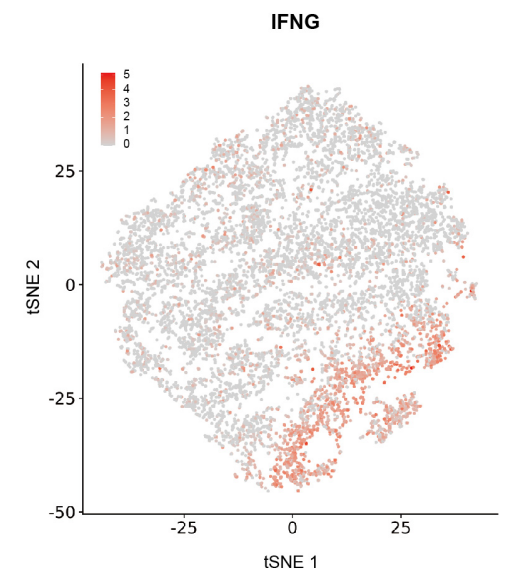

PD1

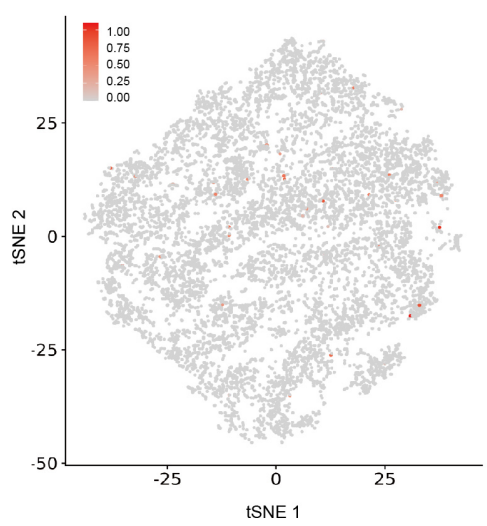


Supplementary figure 11

a

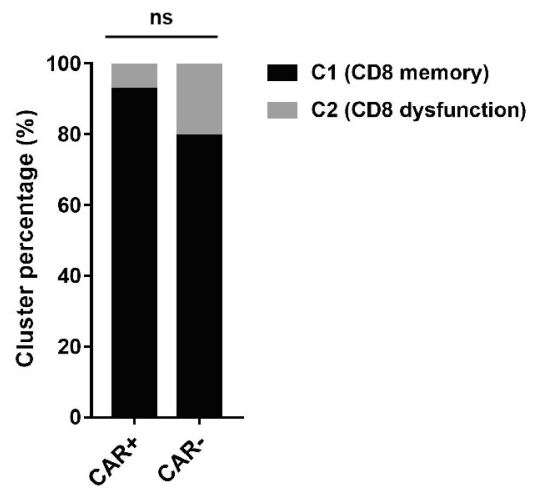

b

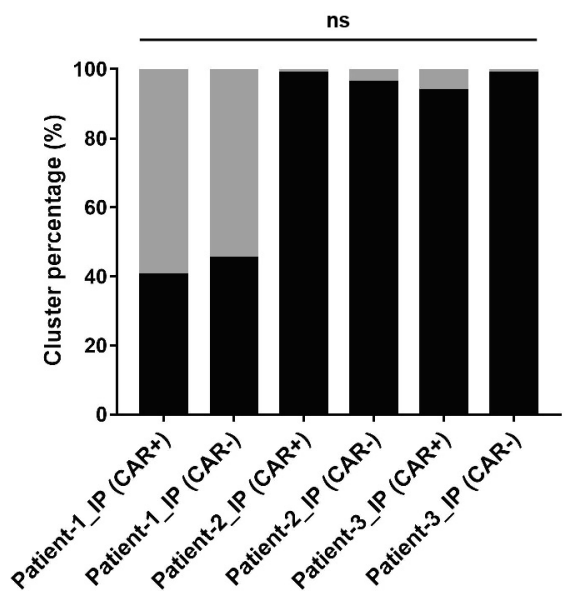

Supplementary figure 11 Proportion of CD8 memory and dysfunction clusters in infusion products

a-b, Comparison of $\mathrm{C} 1$ and $\mathrm{C} 2$ proportion between CAR+ and CAR- cells in mixed (a) and individual (b) samples of infusion products. P values are calculated by two-way ANOVA. 


\section{Supplementary figure 12}

\section{a}

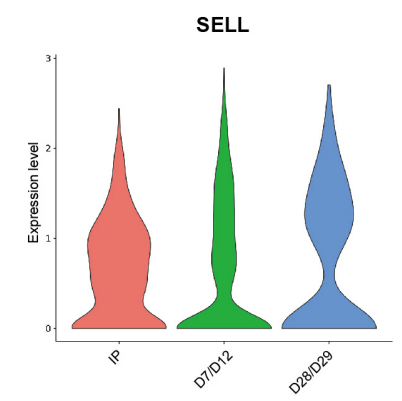

IL7R

LTB
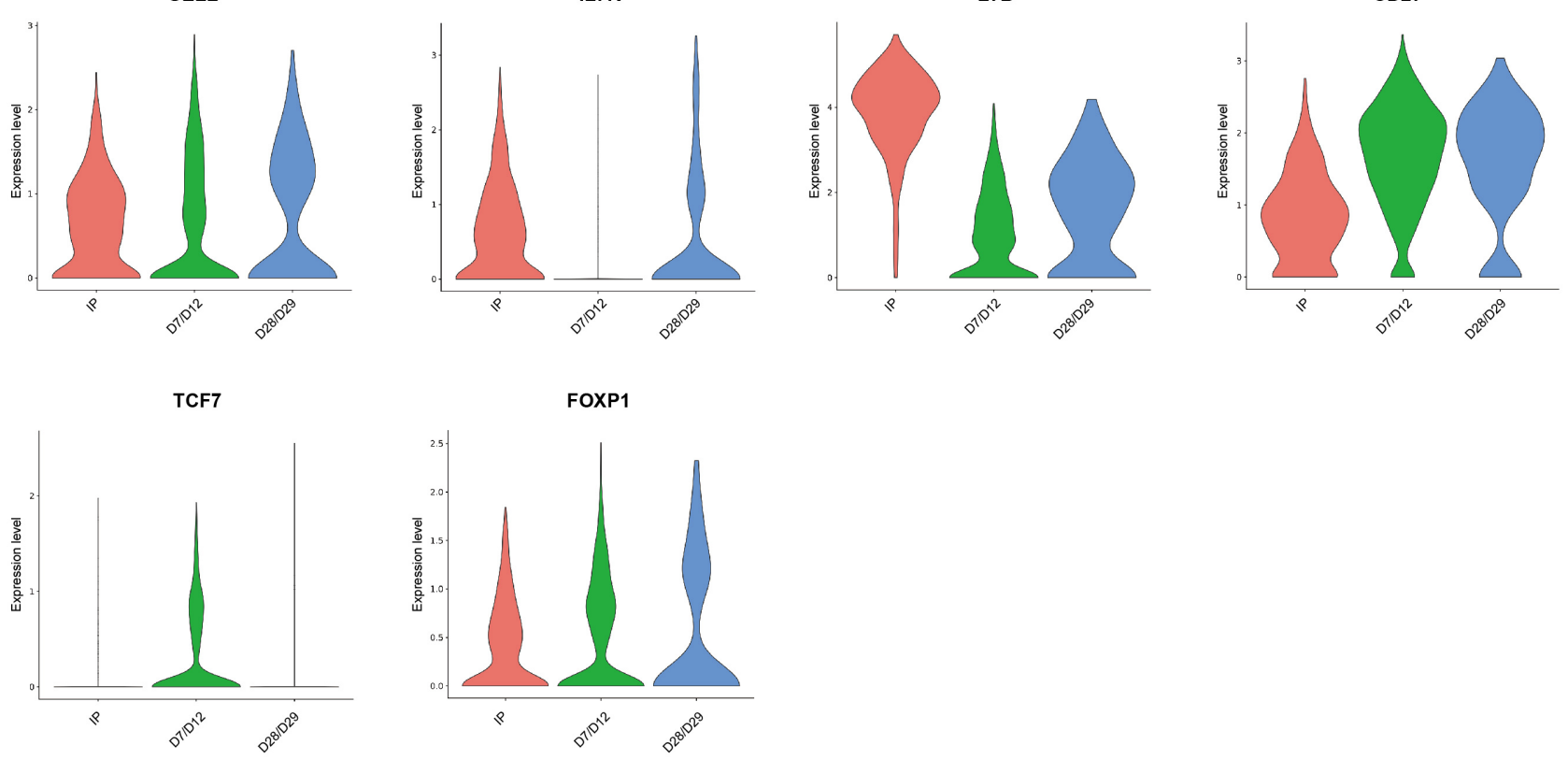

LEF1

LEF1
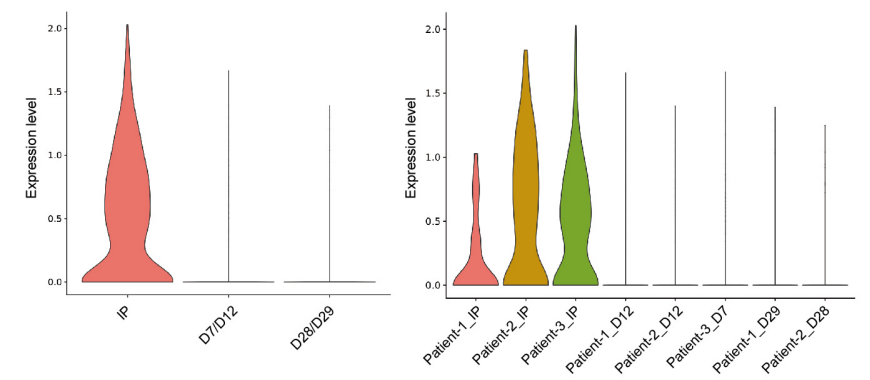

CCR7

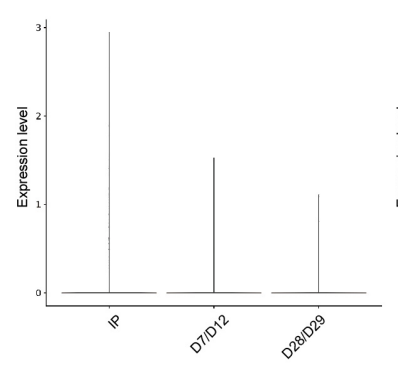

CCR7

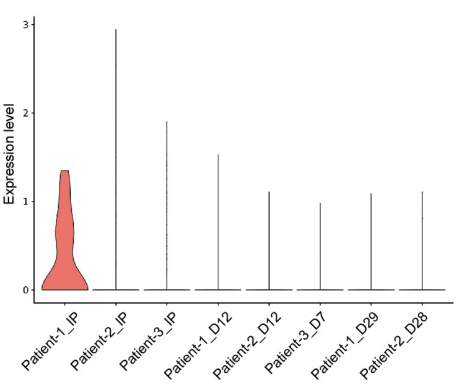

Supplementary figure 12 Expression of CD8 memory genes in non-viral PD1-targeted CAR T cells before and after infusion

$a-b$, Violin plots showing the expression of memory genes in CD8+/CAR+ cells from three patients before and after infusion. Data of mixed (a, b) and individual (b) samples are shown, respectively. The data of patient- 3 sample after 28 days treatment is excluded from mixed samples and not shown individually due to an unreliable low CAR+ cell number. 


\section{Supplementary figure 13}

CTLA4

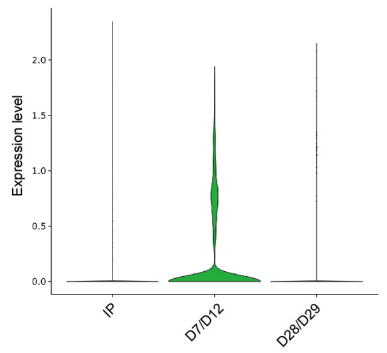

CCL3

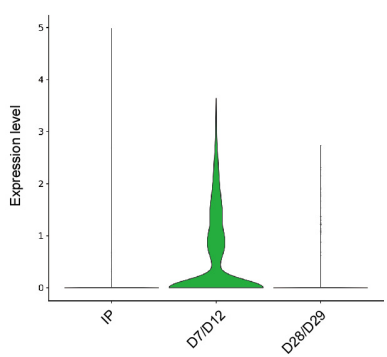

ITGAE

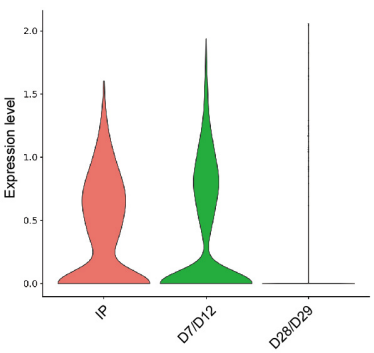

SNAP47

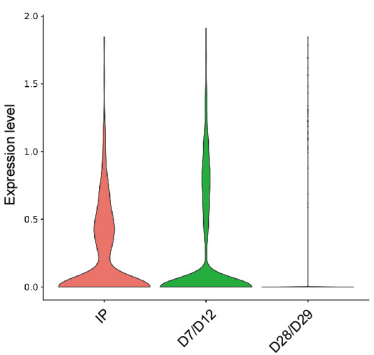

LAG3

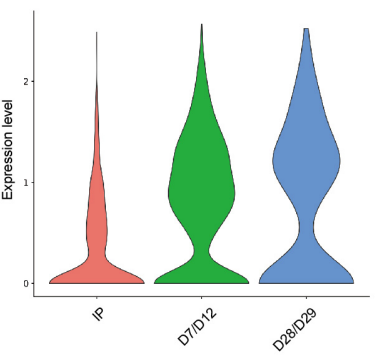

TIGIT
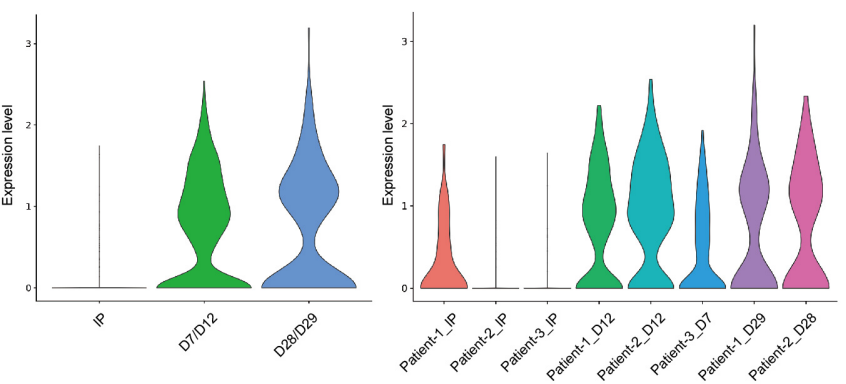

IFNG

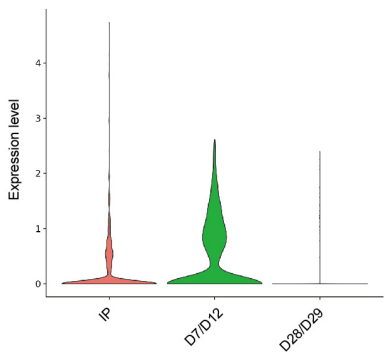

PHLDA1

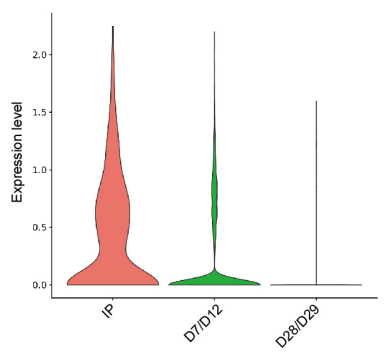

HAVCR2

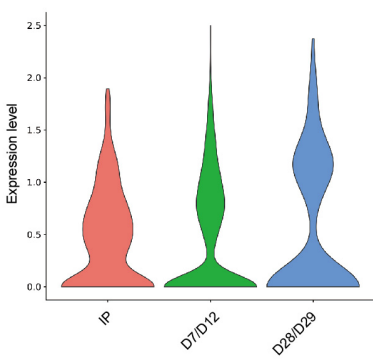

CD38

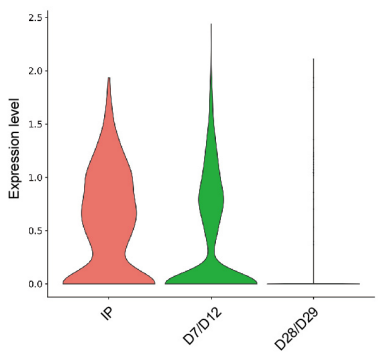

WARS

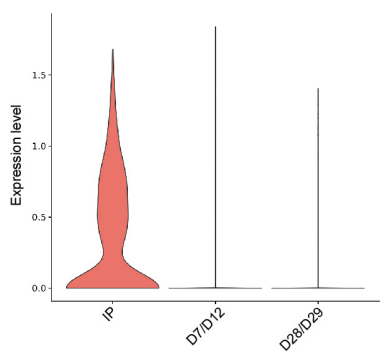

HAVCR2

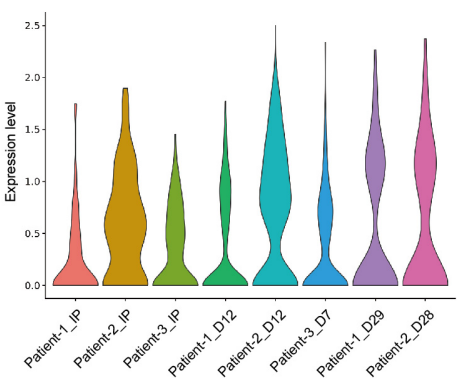

Supplementary figure 13 Expression of CD8 dysfunction genes in non-viral PD1-targeted CAR T cells before and after infusion

$a-b$, Violin plots showing the expression of dysfunction genes in CD8+/CAR+ cells from three patients before and after infusion. Data of mixed (a, b) and individual (b) samples are shown, respectively. The data of patient- 3 sample after 28 days treatment is excluded from mixed samples and not shown individually due to an unreliable low CAR+ cell number. 

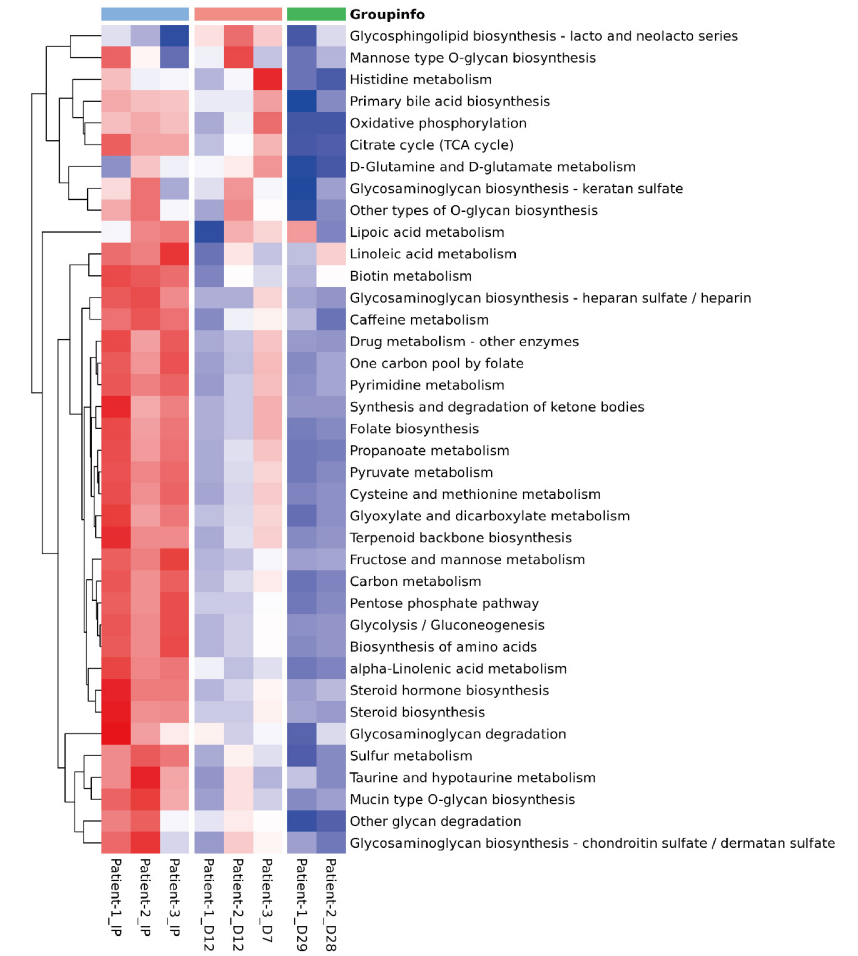

离

Supplementary figure 14 Gene set enrichment analysis in non-viral PD1-integrated CAR T cells before and after infusion

$a-b$, Metabolism (a) and other (b) pathway activities were scored by gene set enrichment analysis (GVSA) using the quantitative set analysis for gene expression (QuSAGE) method in three patient samples before and after infusion. The data of patient- 3 sample after 28 days treatment is excluded from mixed samples and not shown individually due to an unreliable low CAR+ cell number.

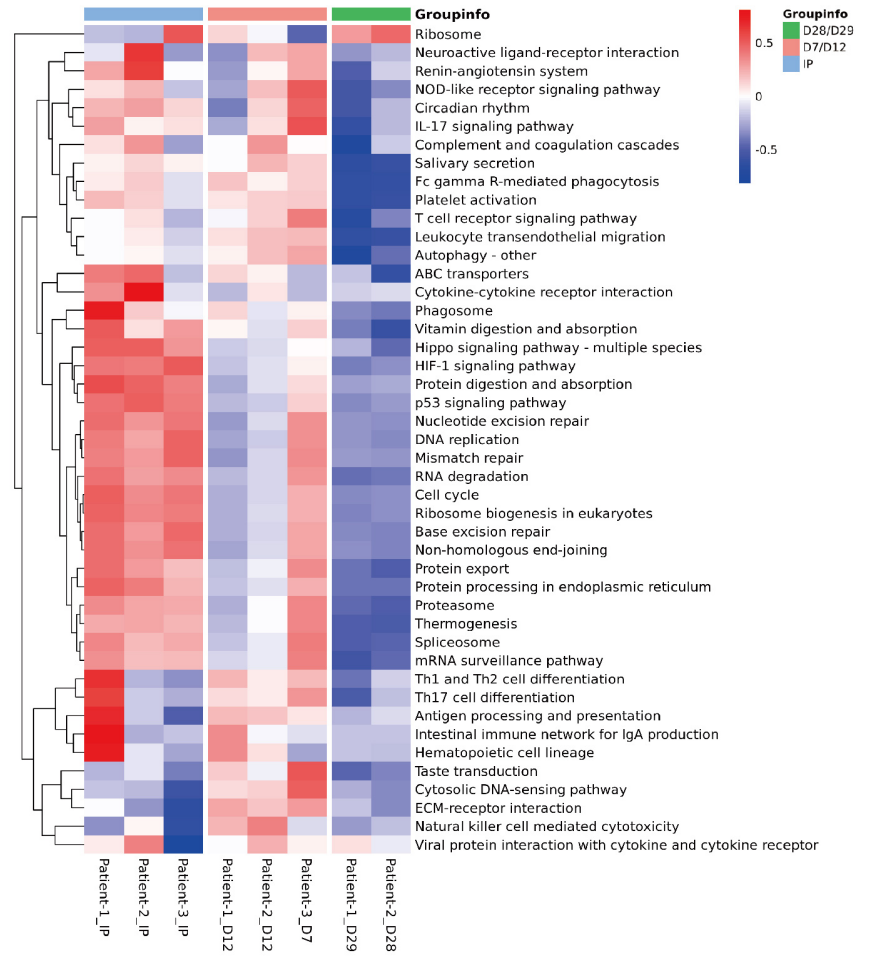

Primary bile acid biosynthesis

Other types of 0 -glycan biosynthesis

Lipoic acid metabolism

Caffeine metabolism

- other enzymes

tion of ketone bodies

Terpenoid backbone biosynthesis

alpha-Linolenic acid metabolism

potaurine metabalism

Glycosaminoglycan biosynthesis - chondroitin sulfate / dermatan sulfate
Groupinfo

L-17 signaling path

rotein expot

cytokine receptor 
Table 1 Patient characteristics, clinical responses and adverse events

\begin{tabular}{|c|c|c|c|c|c|c|c|c|c|c|}
\hline Patient & Sex & $\begin{array}{c}\text { Age } \\
\text { (years) }\end{array}$ & Lymphoma type & $\begin{array}{c}\text { Number of } \\
\text { prior lines of } \\
\text { therapy }^{a}\end{array}$ & $\begin{array}{c}\text { Disease } \\
\text { stage }\end{array}$ & Lymphoma status ${ }^{b}$ & $\begin{array}{c}\text { CAR+ cell dose } \\
(/ \mathrm{kg})\end{array}$ & $\begin{array}{c}\text { Best response } \\
\text { (duration in } \\
\text { months) }\end{array}$ & CRS & $\begin{array}{c}\text { Neurological } \\
\text { toxicity }\end{array}$ \\
\hline Patient-1 & $M$ & 44 & GCB DLBCL & 5 & $\mathrm{~N}$ & Refractory & $0.56 \times 10^{6}$ & $\mathrm{CR}(9+)$ & Grade 1 & None \\
\hline Patient-2 & $M$ & 53 & Non-GCB DLBCL & 4 & N & Refractory & $2.04 \times 10^{6}$ & $\mathrm{CR}(7+)$ & Grade 1 & None \\
\hline Patient-3 & $M$ & 59 & GCB DLBCL & 3 & IV & Refractory & $1.95 \times 10^{6}$ & PR (1) & Grade 1 & None \\
\hline Patient-4 & $M$ & 46 & Non-GCB DLBCL & 10 & $\mathrm{~N}$ & $\begin{array}{l}\text { Relapsed within } 6 \\
\text { months after ASCT }\end{array}$ & $0.8 \times 10^{6}$ & CR $(4+)$ & None & None \\
\hline Patient-5 & $\mathrm{F}$ & 43 & B-LBL & 4 & N & Refractory & $0.76 \times 10^{6}$ & $\mathrm{CR}(4+)$ & None & None \\
\hline Patient-6 & $\mathrm{F}$ & 64 & Non-GCB DLBCL & 4 & $\mathrm{~N}$ & Refractory & $2.35 \times 10^{6}$ & $\mathrm{CR}(3+)$ & Grade 2 & None \\
\hline Patient-7 & $\mathrm{F}$ & 62 & GCB DLBCL & 1 & III & Refractory & $2.15 \times 10^{6}$ & CR $(3.5+)$ & None & None \\
\hline Patient-8 & $\mathrm{F}$ & 51 & $\mathrm{FL}$ & 6 & N & Refractory & $1.9 \times 10^{6}$ & $\mathrm{CR}(3+)$ & None & None \\
\hline
\end{tabular}

ASCT, autologous stem cell transplant; B-LBL, B-cell lymphoblastic lymphoma; CR, complete remission; DLBCL, diffuse large B cell lymphoma; F, female; FL, follicular lymphoma; GCB, germinal center B cell; M, male; PD, progressive disease; PR, partial remission. ${ }^{\mathrm{a} A l l}$ prior lines of therapy for each patient are listed in Supplementary table 4 'Disease was defined as refractory if a patient did not achieve partial or complete remission after the most recent chemotherapy. 'Best response was defined as the best response that a patient achieved after CAR $T$ cell infusion. ${ }^{d}$ Response duration is the time from the first documentation of response, until progression, initiation of off-study treatment or the last documentation of ongoing response. The + symbol indicates an ongoing response. 
Table S1 Deep sequencing analysis of 8 off-target (OT) sites detected by WGS

\begin{tabular}{|c|c|c|c|c|c|c|c|c|c|c|c|}
\hline Number & Chrom & $\begin{array}{c}\text { Predicted } \\
\text { OT pos } \\
\end{array}$ & Indel pos & $\begin{array}{c}\text { Distance } \\
\text { (bp) }\end{array}$ & On-target seq & Predicted OT seq & Strand & Function & Mutation type & $\begin{array}{c}\text { Validated by } \\
\text { deep seq }\end{array}$ & $\begin{array}{c}\text { Nucleotide } \\
\text { repeats }\end{array}$ \\
\hline 1 & $\operatorname{chr} 15$ & 34428733 & 34428916 & 182 & CGACTGGCCAGGGCGCCTGTGGG & CGATAGGCCAGGGCGCCT-GCAG & - & intergenic & $\begin{array}{l}1-5 \text { bp deletion } \\
1 \text { bp insertion }\end{array}$ & Yes & $\begin{array}{l}\text { AAAAAAAAAAAAAAAAA } \\
\text { AAAA }\end{array}$ \\
\hline 2 & $\operatorname{chr} 15$ & 34574943 & 34575106 & 162 & CGACTGGCCAGGGCGCCTGTGGG & CGATAGGCCAGGGCGCCT-GCAG & - & intergenic & NA & No & NA \\
\hline 3 & $\operatorname{chr} 15$ & 34574943 & 34575120 & 176 & CGACTGGCCAGGGCGCCTGTGGG & CGATAGGCCAGGGCGCCT-GCAG & - & intergenic & NA & No & NA \\
\hline 4 & $\operatorname{chr} 17$ & 47703037 & 47702993 & 43 & CGACTGGCCAGGGCGCCTGTGGG & CGAAGGGACAGGGGGTCTGTGAG & + & intronic & 2 bp deletion & Yes & GGGGGGGGG \\
\hline 5 & chr2 & 72911968 & 72912124 & 155 & CGACTGGCCAGGGCGCCTGTGGG & TGAGTGTCTAGGGGGCCTGTAGG & + & intergenic & NA & No & NA \\
\hline 6 & $\operatorname{chr} 21$ & 13246921 & 13246759 & 161 & CGACTGGCCAGGGCGCCTGTGGG & CGTCTAGCCAGGGAGCATCTCAG & - & intergenic & 4 bp insertion & Yes & $\begin{array}{l}\text { TGTGTGTGTGTGTGTGT } \\
\text { GTGTGTGTGTGTGTGTG }\end{array}$ \\
\hline 7 & chr3 & 187139213 & 187139074 & 138 & CGACTGGCCAGGGCGCCTGTGGG & GCAGAGGCCAGGGCGCCGGTAAG & - & intronic & NA & No & NA \\
\hline 8 & chr9 & 131301652 & 131301813 & 160 & CGACTGGCCAGGGCGCCTGTGGG & CGCCTGGCCCGGGAAGCTGTGGG & - & intronic & NA & No & NA \\
\hline
\end{tabular}


Table S2 Deep sequencing analysis of top 29 off-target sites predicted by the Benchling CRISPR tool

\begin{tabular}{|c|c|c|c|c|c|c|}
\hline Site & Chrom & Position & Target sequence & PAM & Strand & Mismatch \\
\hline On-target & $\operatorname{chr} 2$ & 241858825 & CGACTGGCCAGGGCGCCTGT & GGG & + & 0 \\
\hline Off-target 1 & $\operatorname{chr} 12$ & 590629 & CCACTGCCCAGGGCGCCTGG & AAG & + & 3 \\
\hline off-target 2 & $\operatorname{chr} 3$ & 135323926 & CAACTGGCCAGGGCACCTAT & GAG & + & 3 \\
\hline Off-target 3 & $\operatorname{chr} 1$ & 1088609 & CCACCGGCCAGGGCGCCTTT & AAG & + & 3 \\
\hline Off-target 4 & $\operatorname{chr} 8$ & 53878623 & GGACTGGCCAGTGCGGCTGT & $\mathrm{AGG}$ & - & 3 \\
\hline Off-target 5 & $\operatorname{chr} 15$ & 78526890 & AGACTGGCCAGGGAGTCTGT & GAG & - & 3 \\
\hline Off-target 6 & $\operatorname{chr} 3$ & 123344000 & GGACTGGCCAAGGAGCCTGT & AGG & - & 3 \\
\hline Off-target 7 & $\operatorname{chr} 21$ & 43988998 & CGTGTGGCCAGGGGGCCTGT & GAG & + & 3 \\
\hline Off-target 8 & $\operatorname{chr} 11$ & 62855728 & TGCCTGGCCAGGGCGACTGG & CGG & - & 4 \\
\hline Off-target 9 & $\operatorname{chr} 9$ & 92171112 & GGCCTGGCCAGGGCGGCTGG & GGG & - & 4 \\
\hline Off-target 10 & $\operatorname{chr} 11$ & 1153416 & GGCCTGGCCAGGGCCCCTGC & TGG & + & 4 \\
\hline Off-target 11 & $\operatorname{chr} 14$ & 92376556 & GGGCTGGCCAGGGCCCCTGA & GGG & + & 4 \\
\hline Off-target 12 & $\operatorname{chr} 11$ & 36455288 & GGCCTGGCCAGGGAGCCTGG & GAG & + & 4 \\
\hline Off-target 13 & $\operatorname{chr} 1$ & 19454074 & AGAGTGGCCAGGGCTCCTGG & AGG & + & 4 \\
\hline Off-target 14 & $\operatorname{chr} 1$ & 3982141 & TGTCTGGCCAGGGTGCCTGC & $\mathrm{TGG}$ & + & 4 \\
\hline Off-target 15 & $\operatorname{chr} 10$ & 133474998 & AGACAGGCCAGGGCACCTGC & $\mathrm{AGG}$ & - & 4 \\
\hline Off-target 16 & $\operatorname{chr} 13$ & 113104005 & CTGCTGGCCAGGGCGGCTGC & AGG & - & 4 \\
\hline Off-target 17 & $\operatorname{chr} 1$ & 6103274 & AGCCTGGCCAGGGCTCCTTT & GGG & - & 4 \\
\hline Off-target 18 & $\operatorname{chr} 16$ & 53503575 & CAATTGGCCAGGGCGTCTGC & CAG & + & 4 \\
\hline Off-target 19 & $\operatorname{chr} 5$ & 758609 & TGCCTGGCCATGGCGCCTGC & AGG & - & 4 \\
\hline Off-target 20 & $\operatorname{chr} 9$ & 109891067 & CCCCTGGCCAGGGTGCCTGG & AGG & + & 4 \\
\hline Off-target 21 & $\operatorname{chr} 8$ & 11549226 & GGACTGACCAGGGAGCCTGC & AGG & + & 4 \\
\hline 0ff-target 22 & $\operatorname{chr} 2$ & 239056117 & CGCCAGGCCAGGGCGCCAGG & $\mathrm{CAG}$ & + & 4 \\
\hline Off-target 23 & chr2 & 229943631 & CCACGGGCCAGGGCTCCTGA & CAG & - & 4 \\
\hline Off-target 24 & $\operatorname{chr} 10$ & 102415823 & GGACTGGGCAGGGCACCTGG & AGG & - & 4 \\
\hline Off-target 25 & $\operatorname{chr} 7$ & 149765282 & CCCCTGGCCAGCGCGCCTGG & $\mathrm{CGG}$ & - & 4 \\
\hline Off-target 26 & $\operatorname{chr} 6$ & 35078996 & AGGCTGGCCAGGGCTCCAGT & GAG & - & 4 \\
\hline Off-target 27 & $\operatorname{chr} 1$ & 153642303 & CCCCTGGCCAGGGCCCCTAT & GGG & - & 4 \\
\hline Off-target 28 & $\operatorname{chr} 2$ & 201929252 & CCACAGGCCAGGGTGCCTGG & $\mathrm{AAG}$ & + & 4 \\
\hline Off-target 29 & $\operatorname{chr} 5$ & 1104177 & TGACTGCCCAGGGCGTCTCT & GAG & + & 4 \\
\hline
\end{tabular}


Table S3 Infusion products of non-viral PD1 integrated CAR T cells

\section{Patient Weight $(\mathrm{kg}) \quad$ CAR+ cell dose $(/ \mathrm{kg}) \quad$ Total cell number $\quad$ CAR+ $(\%)$ Viability $(\%) \quad$ CD4/CD8 ratio}

\begin{tabular}{lcccccc}
\hline Patient-1 & 62 & $0.56 \times 10^{6}$ & $6.32 \times 10^{8}$ & 5.49 & 96.2 & 0.75 \\
Patient-2 & 89 & $2.04 \times 10^{6}$ & $9.41 \times 10^{8}$ & 19.3 & 97.5 & 0.15 \\
Patient-3 & 75 & $1.95 \times 10^{6}$ & $8.55 \times 10^{8}$ & 17.1 & 98.6 & 0.35 \\
Patient-4 & 70 & $0.8 \times 10^{6}$ & $1.15 \times 10^{9}$ & 4.87 & 95.2 & 1.11 \\
Patient-5 & 63 & $0.76 \times 10^{6}$ & $1.01 \times 10^{8}$ & 47.3 & 93.4 & 0.01 \\
Patient-6 & 60 & $2.35 \times 10^{6}$ & $5.90 \times 10^{8}$ & 23.9 & 92.2 & 0.50 \\
Patient-7 & 59 & $2.15 \times 10^{6}$ & $7.17 \times 10^{8}$ & 17.7 & 93.2 & 0.18 \\
Patient-8 & 42 & $1.9 \times 10^{6}$ & $5.12 \times 10^{8}$ & 15.6 & 97.4 & 0.30 \\
\hline
\end{tabular}


Table S4 Prior lines of therapy for each patient

\begin{tabular}{|c|c|c|}
\hline Patient & $\begin{array}{l}\text { Number of prior } \\
\text { lines of therapy }\end{array}$ & Prior therapies \\
\hline Patient-1 & 5 & $\begin{array}{c}\text { R-CHOP } \\
\text { Sintilimab + ICE + R-Gemox + Chidamide } \\
\text { R-Gemox + Chidamide } \\
\text { Radiation therapy } \\
\text { Rituximab + Lenalidomide + Chidamide }\end{array}$ \\
\hline Patient-2 & 4 & $\begin{array}{c}\text { R-COP } \\
\text { RCD } \\
\text { Lenalidomide } \\
\text { R-DA-EPOCH }\end{array}$ \\
\hline Patient-3 & 3 & $\begin{array}{l}\text { R-CHOP } \\
\text { R2-CHOP } \\
\text { R-CHOPE }\end{array}$ \\
\hline Patient-4 & 10 & $\begin{array}{c}\text { R2-CHOP } \\
\text { Rituximab + Lenalidomide } \\
\text { Rituximab } \\
\text { Rituximab+ lbrutinib+ EPOCH } \\
\text { R-Hyper-CVADA } \\
\text { Rituximab+ Ibrutinib+ Hyper-CVADA } \\
\text { Rituximab+ lbrutinib } \\
\text { ASCT } \\
\text { SMART } \\
\text { Brentuximab vedotin + Ibrutinib + CHOP }\end{array}$ \\
\hline Patient-5 & 4 & 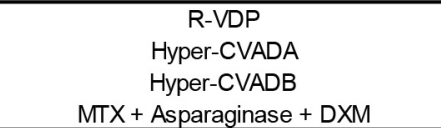 \\
\hline Patient-6 & 4 & $\begin{array}{c}\text { R-CHOP } \\
\text { R-GDP } \\
\text { R-CHOP } \\
\text { R2-DA-EPOCH }\end{array}$ \\
\hline Patient-7 & 1 & $\mathrm{R}-\mathrm{CHOP}$ \\
\hline Patient-8 & 6 & $\begin{array}{c}\text { BR C1 } \\
\text { BR C2 } \\
\text { BR C3 } \\
\text { BR C4 } \\
\text { R2-CHOP } \\
\text { R-GDP }\end{array}$ \\
\hline
\end{tabular}

ASCT: autologous stem cell transplant; BR: bendamustine, rituximab; CHOP: cyclophosphamide, adriamycin, vincristine, prednisone; $\mathrm{CHOPE:} \mathrm{cyclophosphamide,} \mathrm{adriamycin,} \mathrm{vincristine,}$ prednisone, etoposide; COP: cyclophosphamide, vincristine, prednisone; DA: dose adjustment; DXM: dexamethasone; EPOCH: etoposide, prednisone, vincristine, cyclophosphamide, doxorubicin; GDP: gemcitabine, dexamethasone, cisplatin; Hyper-CVADA: cyclophosphamide, vincristine, doxorubicin, dexamethasone; Hyper-CVADB: methetrexate, cytarabine; ICE: ifosfamide, carboplatin, etoposide; MTX: methotrexate; R: rituximab; R2: revlimid, rituximab; RCD: rituximab, cyclophosphamide, dexamethasone; R-GemOx: rituximab, gemcitabine, oxaliplatin; SMART: simultaneous modulated accelerated radiotherapy; VDP: vincristine, daunorubicin, prednisone. 
Table S5 Summary of adverse events in the study

\begin{tabular}{|c|c|c|c|c|}
\hline AE category & Toxicity & All grades & Grade 1/2 & Grade $3 / 4$ \\
\hline CRS & I & 4 & 4 & 0 \\
\hline \multirow{8}{*}{ Hematologic } & Decrease in white blood cells & 17 & 9 & 8 \\
\hline & Increase in white blood cells & 1 & 1 & 0 \\
\hline & Decrease in lymphocytes & 12 & 1 & 11 \\
\hline & Decrease in platelet & 5 & 1 & 4 \\
\hline & Decrease in neutrophil & 19 & 5 & 14 \\
\hline & Hypoalbuminemia & 2 & 2 & 0 \\
\hline & Anaemia & 5 & 1 & 4 \\
\hline & Low fibrinogen & 1 & 1 & 0 \\
\hline \multirow{2}{*}{ Electrolyte } & Hypocalcemia & 4 & 4 & 0 \\
\hline & Alkaline phosphatase & 1 & 1 & 0 \\
\hline Metabolic & Hypertriglyceridemia & 4 & 4 & 0 \\
\hline \multirow{6}{*}{ Gastrointestinal } & Abdominal distention & 1 & 1 & 0 \\
\hline & Abdominal distention and nausea & 1 & 1 & 0 \\
\hline & Nausea & 1 & 1 & 0 \\
\hline & Dysphagia & 1 & 1 & 0 \\
\hline & Throat discomfort & 2 & 2 & 0 \\
\hline & Abdominal aching & 2 & 2 & 0 \\
\hline \multirow{2}{*}{ Respiratory } & Cough or with expectoration & 2 & 2 & 0 \\
\hline & Thick breathing sounds & 1 & 1 & 0 \\
\hline \multirow{2}{*}{ Neurologic } & Headache & 1 & 1 & 0 \\
\hline & Dizzy & 1 & 1 & 0 \\
\hline \multirow{5}{*}{ Other } & Fatigue & 1 & 1 & 0 \\
\hline & Multiple lumps & 1 & 1 & 0 \\
\hline & Small amount of urine & 1 & 1 & 0 \\
\hline & Left leg swell & 1 & 1 & 0 \\
\hline & Subcutaneous mass of left elbow & 1 & 1 & 0 \\
\hline \multicolumn{2}{|r|}{ Total } & 93 & 52 & 41 \\
\hline
\end{tabular}


Figures

a

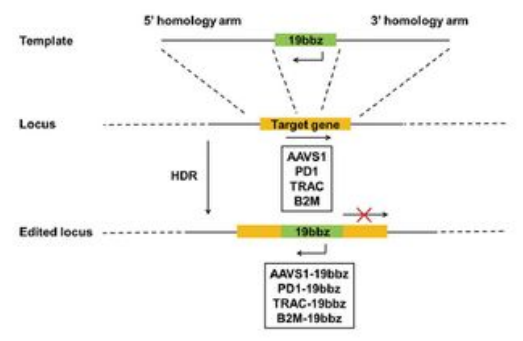

e $f$ b

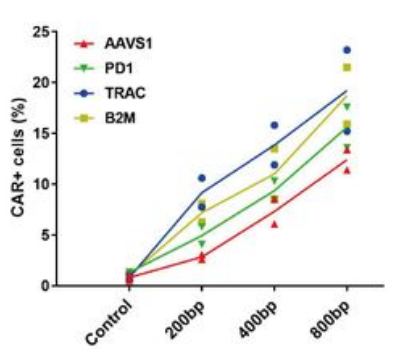

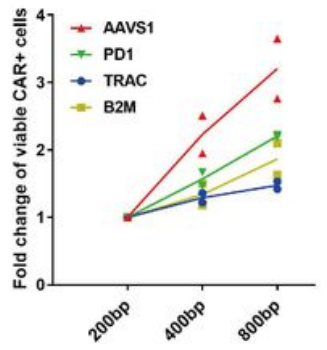

d

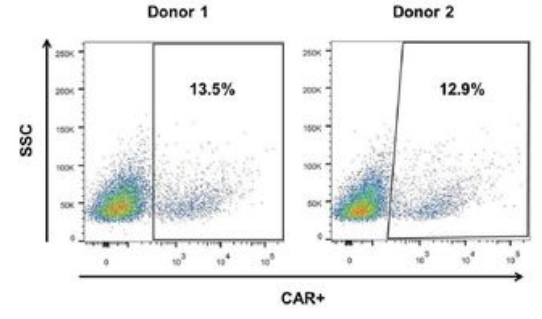

g

CD69

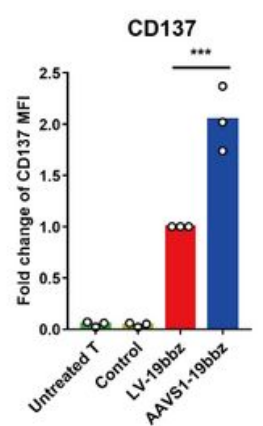

i

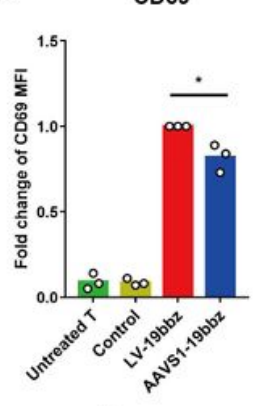

PD1

LAG3 h

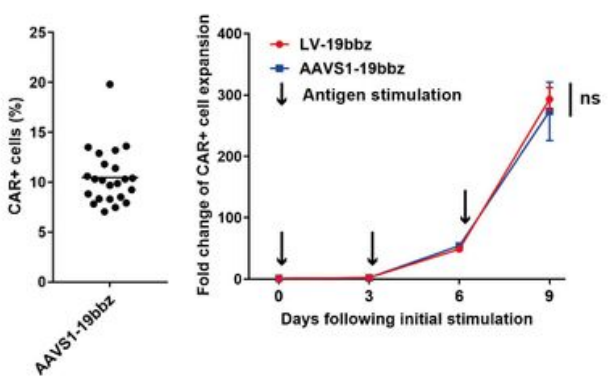

TNF- $\alpha$

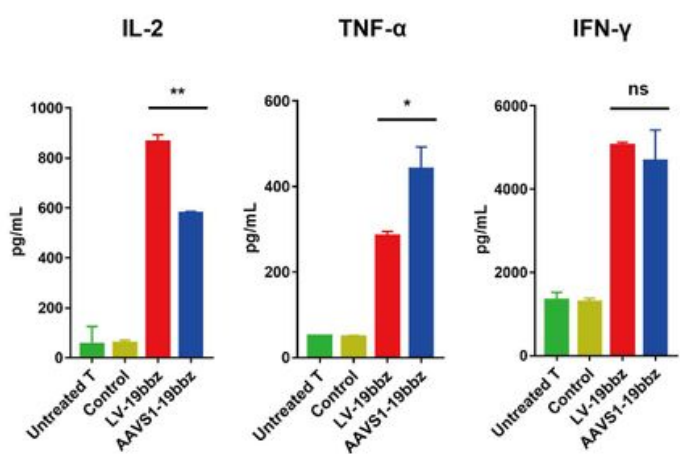

IFN-Y
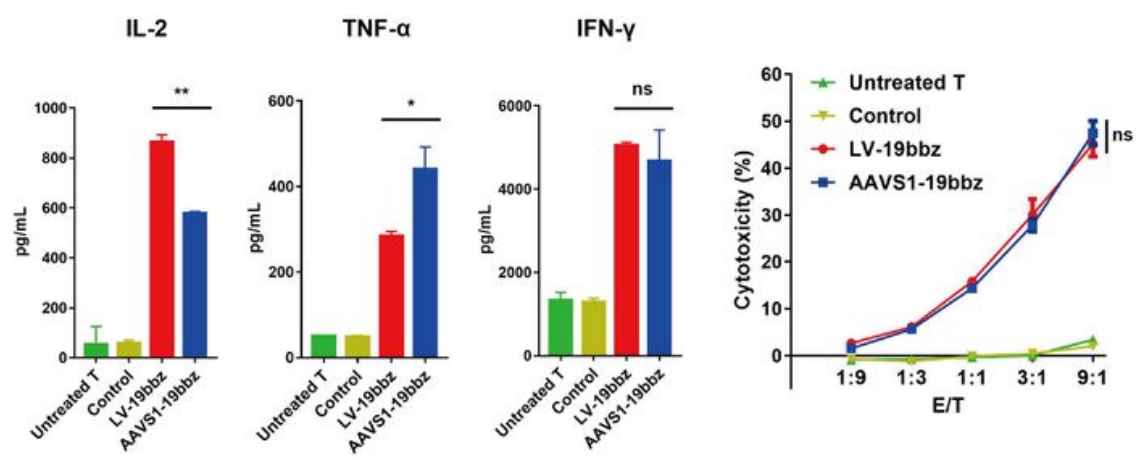
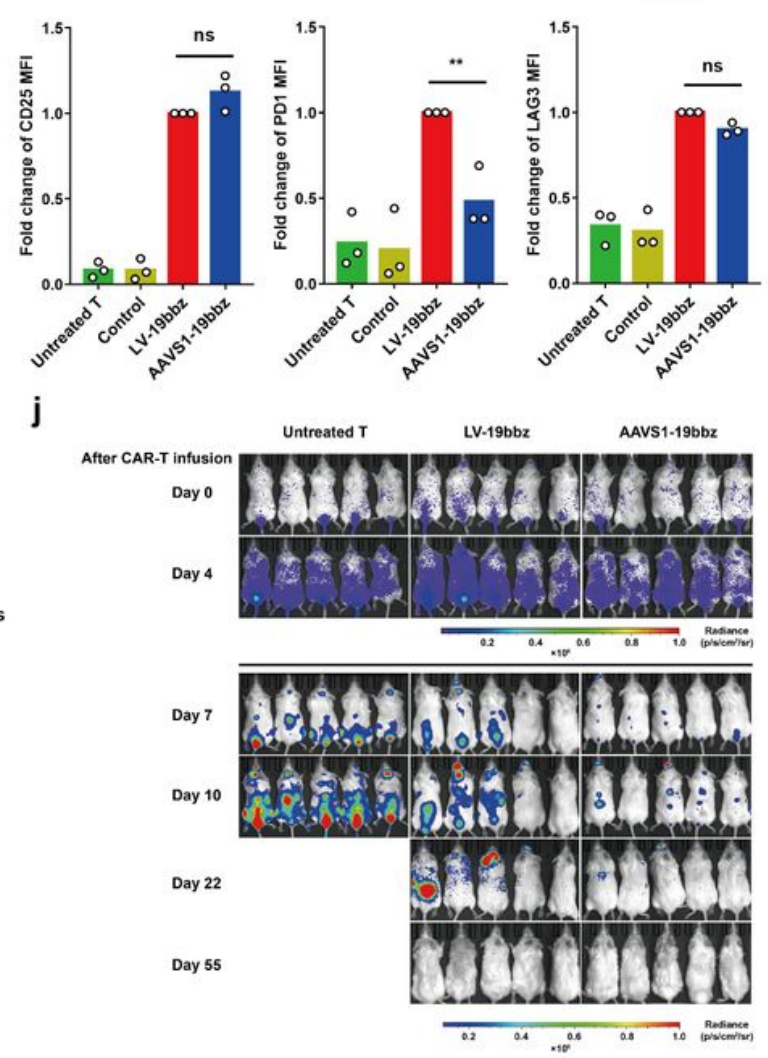

Figure 1

Non-viral AAVS1-integrated CAR T cells eliminate tumor cells as effectively as conventional CAR T cells (see Manuscript file for full figure legend) 
a

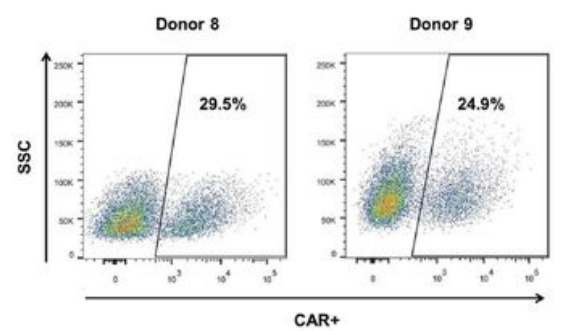

b

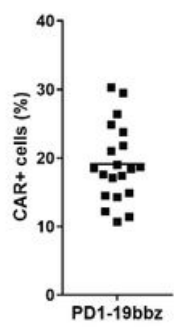

C

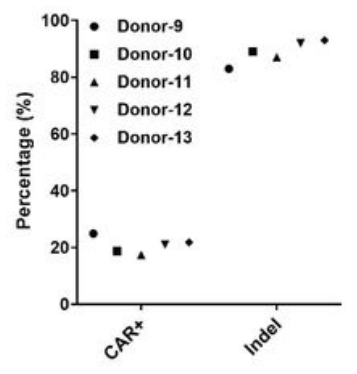

d

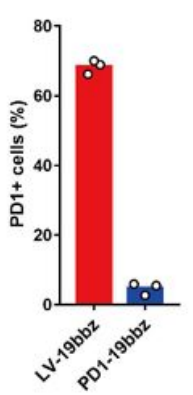

e

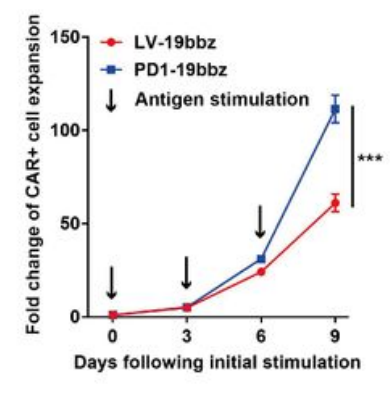

h
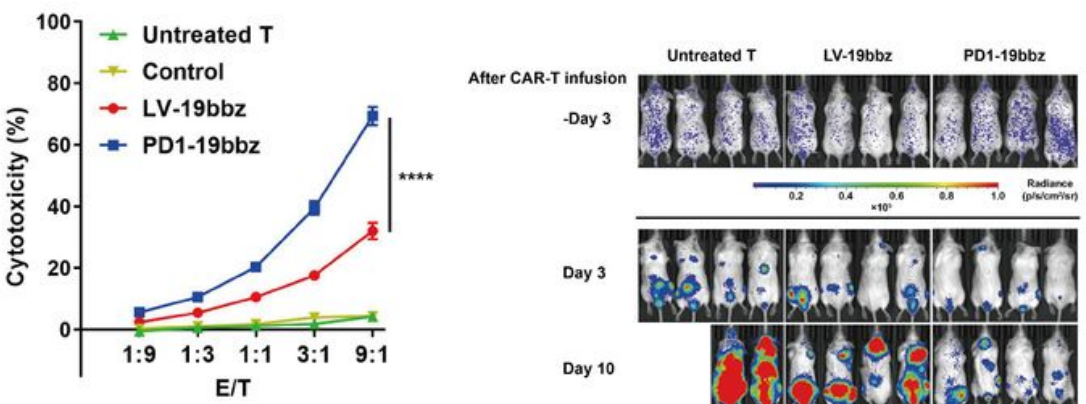

Day 3

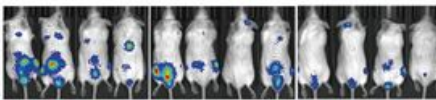

Day 10

Day 14

i

TNF- $\alpha$

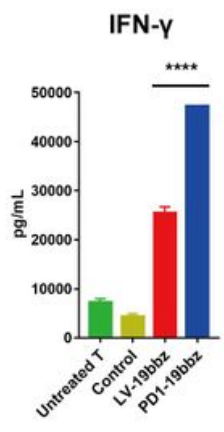

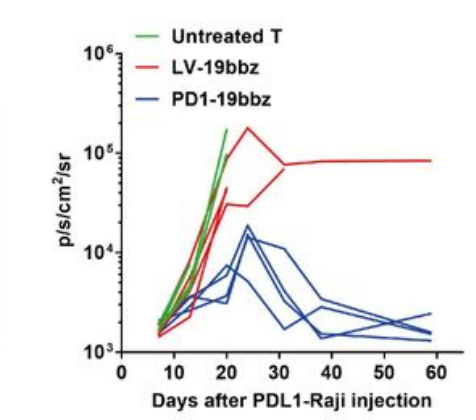

IL-2

g

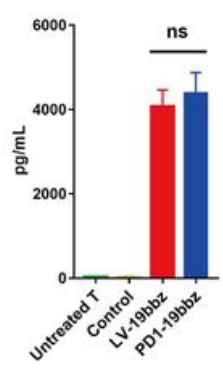

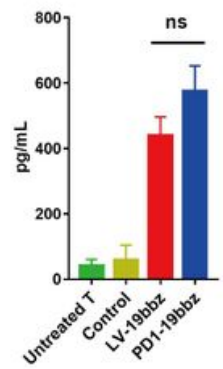

Day 21

Day 28

Day 49
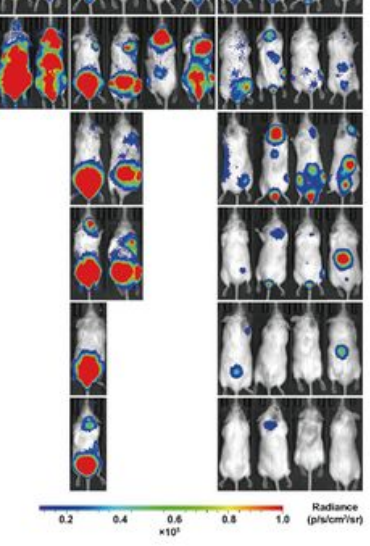

Figure 2

Non-viral PD1-integrated CAR T cells outperform conventional CAR T cells (see Manuscript file for full figure legend) 
a

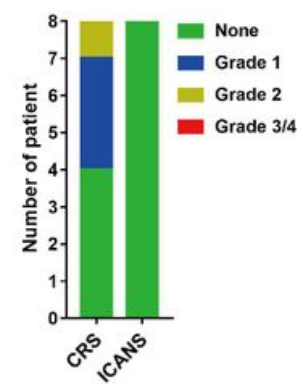

d

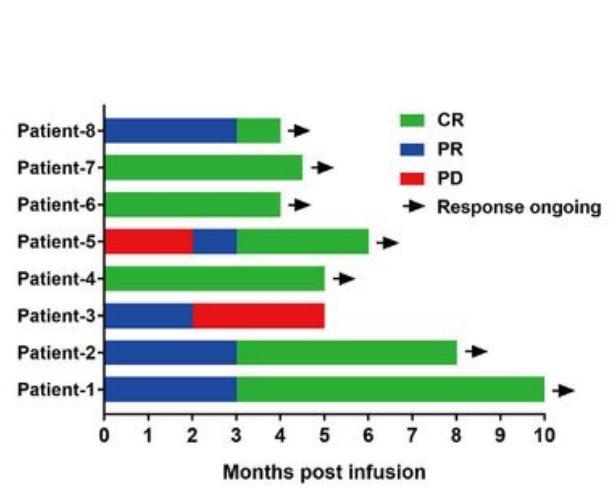

C
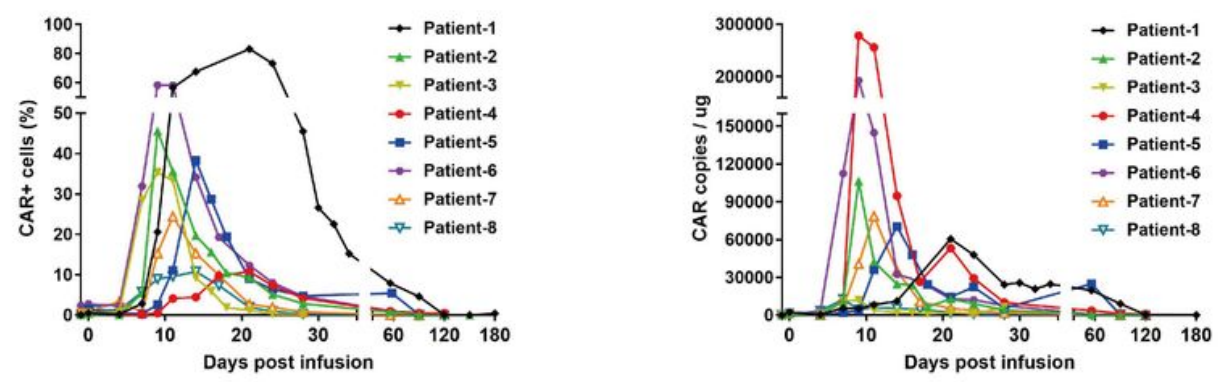

e

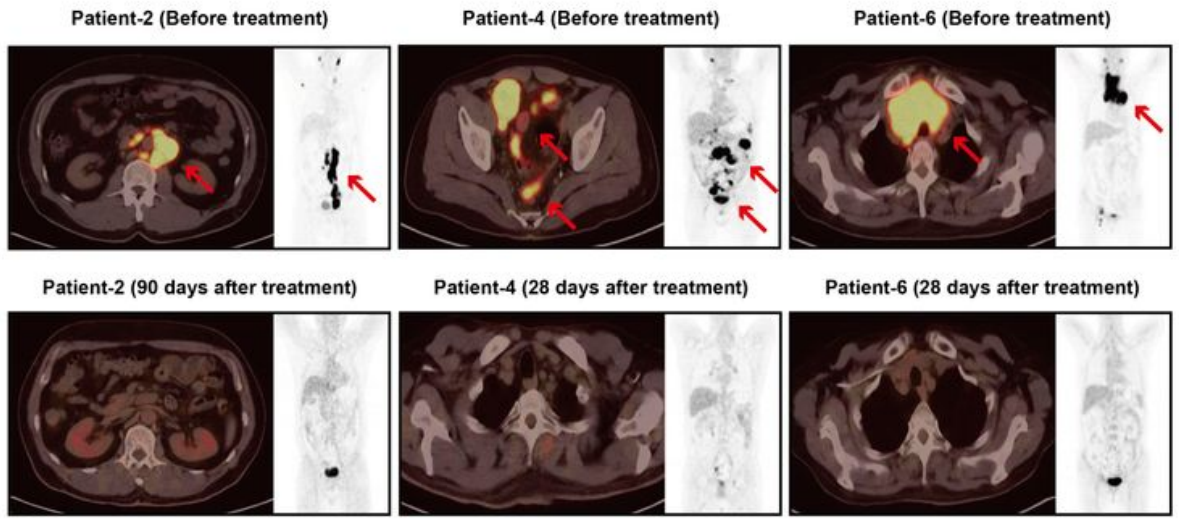

Figure 3

Non-viral PD1-integrated CAR T cells potently eliminate tumor cells in patients with r/r B-NHL without serious toxicity (see Manuscript file for full figure legend) 
a

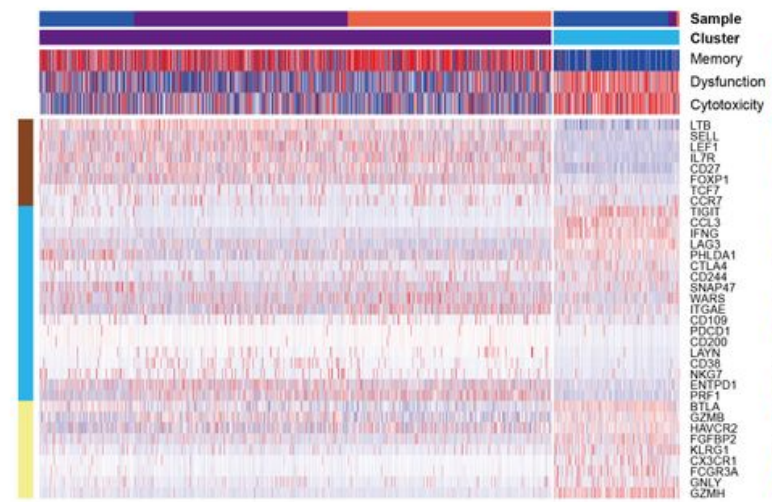

b

C
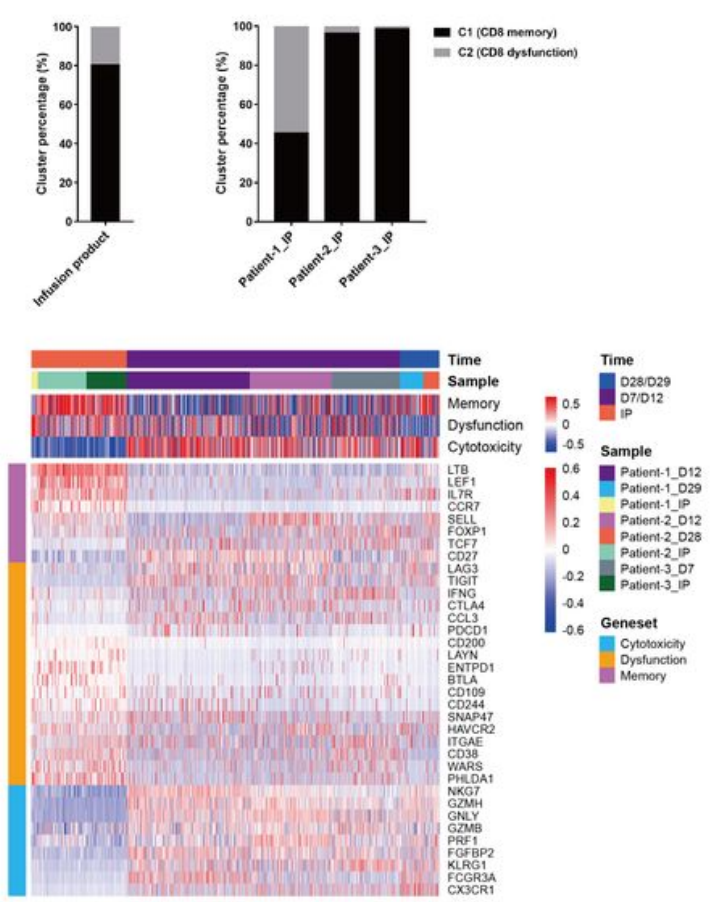

d

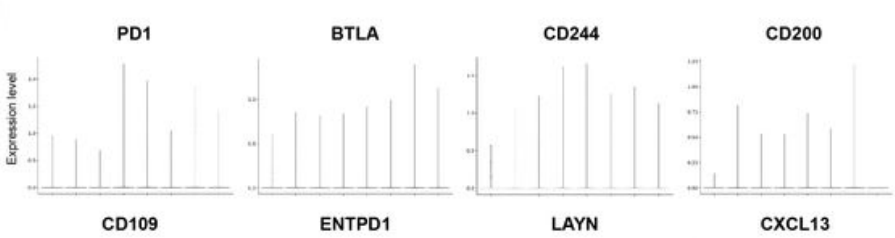

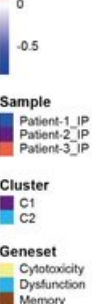

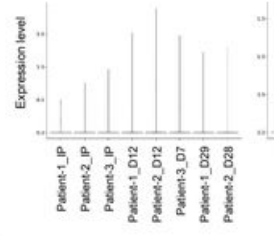

SELL
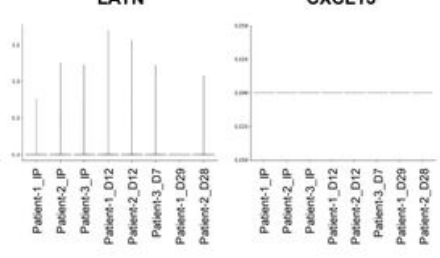

e
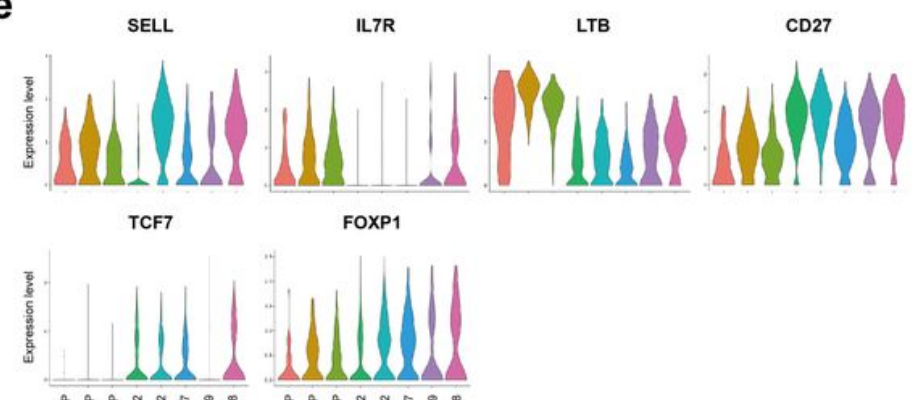

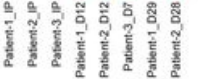

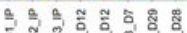

f
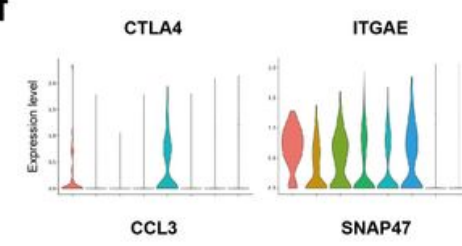

.

IFNG

CD38

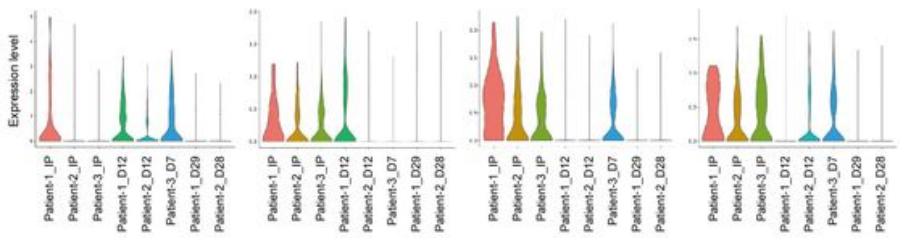

Figure 4

Single-cell RNA sequencing of non-viral PD1-integrated CAR T cells before and after infusion (see Manuscript file for full figure legend)

\section{Supplementary Files}

This is a list of supplementary files associated with this preprint. Click to download.

- TablesS1S5.pdf

- TableS6.xIsx

- Tables7.xIsx

- Tables8.xlsx

- Tables9.xlsx 